
6

\title{
Thin film drainage dynamics of wheat and rye dough liquors and
}

\section{oat batter liquor}

Frederik Janssen ${ }^{1}$, Arno G.B. Wouters ${ }^{1}$, Emmanouil Chatzigiannakis ${ }^{2}$,

Jan A. Delcour ${ }^{1}$, and Jan Vermant ${ }^{2}$

${ }^{1}$ Laboratory of Food Chemistry and Biochemistry and Leuven Food Science and Nutrition Research Centre (LFoRCe), KU Leuven, Kasteelpark Arenberg 20, B-3001 Leuven, Belgium

${ }^{2}$ Laboratory of Soft Materials, Department of Materials, ETH Zürich, Vladimir-Prelog-Weg 5, CH-8093 Zurich, Switzerland

3
\section{(1)} 8

*Corresponding author: Frederik Janssen

Phone: +32 (0) 16374224

Fax: +32(0) 16321997

E-mail address: Frederik.Janssen@kuleuven.be 


\section{Abstract}

To obtain bread with a high loaf volume and homogeneous crumb, gas cells require sufficient stability until the discontinuous gas structure of dough is transformed into the continuous structure of bread. At the end of fermentation, dough gas cells are supposedly regionally separated by two air-water (AW) interfaces and a liquid film. 'Dough liquor' (DL) or 'batter liquor' (BL), i.e. the supernatant after ultracentrifugation of dough and batter, respectively, is a model system for the fluid in these films. We report here on drainage dynamics of free-standing thin films (TFs) prepared from wheat and rye DLs and oat BL after applying a driving pressure of either 50 or $200 \mathrm{~Pa}$ at bulk concentrations lower than and equal to ('native') those in the respective supernatants obtained by ultracentrifugation. At lowered bulk concentrations, wheat and rye DL TFs were surrounded by planar and dimpled A-W interfaces, respectively, both of which were composed of weakly viscoelastic mixed protein-lipid films. In contrast, lipids stabilized dimpled oat BL TFs by migrating along their A-W interfaces and thus by generating Marangoni stresses. Liquid drainage and subsequent film rupture occurred rapidly in these TFs. At native bulk concentrations, TFs generally drained much slower and they were even found to be stable at small thicknesses $(<100 \mathrm{~nm})$. Here, lipids stabilized dimpled wheat DL and oat BL TF A-W interfaces by Marangoni effects or by forming a densely-packed layer, respectively. In addition, wheat DL and oat BL TFs at their native concentrations exhibited stratification due to layered structuring of micelles, which contributed to TF stability by exerting oscillatory structural forces. Thus, it can here be concluded that wheat $\mathrm{DL}$ and oat $\mathrm{BL}$ constituents at their native concentrations may contribute substantially to stabilizing gas cells in dough. 


\section{Introduction}

Bread loaf volume and crumb structure depend on the total number and size distribution of gas cells incorporated during dough formation and on how they are stabilized during dough fermentation and baking. In wheat bread making, the viscoelastic gluten protein network provides gas cells with structural support (van Vliet, Janssen, Bloksma and Walstra, 1992). It has been proposed that this network locally ruptures as a result of its substantial extension upon dough expansion in the late stages of fermentation and thus fails to enclose some gas cell areas (Gan et al. 1990). However, the extent to which dough films in between gas cells are ruptured likely depends on the proofing time. Nonetheless, from the moment such rupture occurs gas cells are believed to be stabilized only by a liquid film containing bulk [i.e. amongst others, non-starch polysaccharides (NSPs)] and surface-active (i.e. proteins and lipids) constituents (Gan, Ellis and Schofield, 1995). Over the past two decades, further evidence has been brought forward to support this concept (Dubreil, Biswas and Marion, 2002; Li, Dobraszczyk and Wilde, 2004; Turbin-Orger et al. 2015a). It thus seems that at the end of fermentation at least some gas cells in dough are separated only by a liquid film enclosed by two airwater $(A-W)$ interfaces.

The liquid film constituents are believed to be part of the aqueous phase of dough. At least a certain fraction of this phase can be isolated from wheat dough by ultracentrifugation (Baker, Parker and Mize, 1946; Mauritzen and Stewart, 1965; MacRitchie, 1976; Larsson and Eliasson, 1996). Noteworthy is that the total amount of DL recovered by ultracentrifugation strongly depends on the total dough water content (Larsson and Eliasson, 1996; Sahi, 2003) and even linearly increases above a certain wheat dough water absorption (Larsson and Eliasson, 1996). The obtained supernatant is generally referred to as 'dough liquor' (DL) and has long been used as a model system for investigating the properties of wheat dough aqueous phase constituents. Most research in this regard indeed relates to DL isolated from wheat dough (MacRitchie, 1976; Sahi, 1994; Mills, Wilde, Salt and Skeggs, 2003; Sahi, 2003; Primo-Martín, Hamer and de Jongh, 2006; Salt et al. 2006; Pauly, Pareyt, Fierens and 
Delcour, 2014; Turbin-Orger et al. 2015b; Salt et al. 2018; Melis, 2019). Today's consumers are interested in consuming a variety of bread products. Examples of such product types are non-wheat breads. However, non-wheat breads often have poorer loaf volume and crumb structure than wheat bread because they lack the typical wheat gluten network in their doughs or batters. Hence, it can be reasoned that the presumed mechanism of gas cell stabilization by dough or batter aqueous phase constituents may be even of greater importance in non-wheat than in wheat bread making. In recent work from our group, the composition and A-W interfacial characteristics of wheat, rye and oat DLs were investigated in-depth (Janssen, Wouters, Pauly and Delcour, 2018; Janssen et al. 2020a; Janssen et al. 2020c). Briefly, it was concluded that wheat DL stabilized A-W interfaces are composed of a weakly viscoelastic mixed protein-lipid film. Moreover, it was found that arabinoxylan (AX), the most abundant NSP in wheat flour, weakens the adsorbed wheat DL film by interacting with its constituents (Janssen et al. 2020a). Rye DL constituents form a strongly viscoelastic protein-dominated interfacial film (although some lipids were also present), which is further strengthened in the presence of AX. At oat DL stabilized A-W interfaces, a lipid-dominated film not affected by $\beta$-D-glucan, the major NSP in oat flour, is present (Janssen et al. 2020a). Despite this in-depth characterization of the A-W interfacial properties of these DLs, it remains difficult to directly relate these findings to bread loaf volume or crumb characteristics. It should be mentioned that in these studies (Janssen et al. 2018; Janssen et al. 2020a; Janssen et al. 2020c) as well as in previous reports in literature (MacRitchie, 1976; Sahi, 1994; Mills et al. 2003; Sahi, 2003; Primo-Martín et al. 2006; Salt et al. 2006; Pauly et al. 2014; Turbin-Orger et al. 2015b; Salt et al. 2018; Melis, 2019), A-W interfacial measurements were conducted using DLs at significantly lower concentrations than those occurring in the supernatant after ultracentrifugation. This mostly relates to the fact that the accuracy and sensitivity of the used interfacial dilatational and shear rheological measurements considerably decrease at higher sample bulk phase viscosities $\left(\eta_{b}\right)$. Moreover, whether a foam is stable or not does not merely depend on the composition of the A-W interfaces surrounding the gas cells but also on the rate at which liquid drains from the thin films (TFs) between these A-W interfaces. In food science, the focus of free-standing TF drainage studies 
has been on isolated proteins such as casein (Marinova et al. 1997; Dimitrova, Leal-Calderon, Gurkov and Campbell, 2001; Cascão Pereira, Johansson, Radke and Blanch, 2003; Marinova et al. 2014; Chen et al. 2017; Chen et al. 2018; Obeid et al. 2019), bovine serum albumin (Marinova et al. 1997; Dimitrova et al. 2001; Cascão Pereira et al. 2003; Marinova et al. 2014; Lin et al. 2018), $\beta$-lactoglobulin (Dimitrova et al. 2001; Basheva, Gurkov, Christov and Campbell, 2006; Rullier, Axelos, Langevin and Novales, 2009, 2010; Gochev, Retzlaff, Exerowa and Miller, 2014; Gochev et al. 2020), and whey proteins (Marinova et al. 2009). TFs separated by mixed protein-surfactant stabilized A-W interfaces have also been studied (Wilde, Rodriguez niño, Clark and Rodriguez Patino, 1997; Husband and Wilde 1998; Sarker, Wilde and Clark, 1998; Maldonado-Valderrama and Langevin, 2008). Last but not least, Senée, Robillard and Vignes-Adler (1999) have studied the drainage of TFs from Champagnes.

In most of the above studies, the focus has been on TFs at equilibrium. This means that the TF is slowly drained until the disjoining pressure $(\Pi)$ equals the capillary hydrostatic pressure $\left(P_{c}\right)$ (Bergeron and Radke, 1992). In this manner, the behavior at equilibrium is studied. However, this approach does not allow assessing the dynamics of the TF drainage process and the role of hydrodynamic forces in particular. However, these dynamics come into play when gas cells approach each other and even to a large extent determine when TF rupture and, subsequently, coalescence occur (Dagastine et al. 2006; Vakarelski et al. 2010; Chatzigiannakis, Veenstra, ten Bosch and Vermant, 2020).

At large TF thickness (>100 $\mathrm{nm}$ ), the disjoining pressure $(\Pi)$ is negligible and the rate at which liquid drains from a TF between two A-W interfaces under an applied $P_{c}$ depends on the:

1. capillarity and hydrodynamics (Fig $1 \mathrm{~A}$ ). Applying a $P_{c}$ may deform the A-W interfaces of a TF into a so-called 'dimple'. This implies a concave shape of the A-W interfaces surrounding the TF when looked at from within. Dimpled A-W interfaces are the result of an increased hydrodynamic pressure $\left(P_{H}\right)$ in the center of the TF (Frankel and Mysels, 1962; Ivanov and Kralchevsky, 1997). The pronounced curvature of a dimpled TF decreases $P_{c}$. This results in a reduced rate of liquid drainage as compared to that from between two planar interfaces (Joye, Hirasaki and Miller, 1992). As liquid drainage proceeds in such system, the dimple may be 
'washed out' from the TF, leading to two planar A-W interfaces (although small thickness corrugations are often observed) (Joye, Hirasaki and Miller, 1994; Kannan, Shieh, Leiske and Fuller, 2018). This washing out process is believed to be a surface tension ( $\sigma$ ) driven hydrodynamic instability which accelerates liquid drainage and is therefore considered detrimental for TF stability (Joye et al. 1994).

2. stress-boundary conditions (Fig 1B). The rate at which liquid drains from a system depends on the gradient of the velocity field at the natural boundaries of the flow domain, in this case the A-W interfaces surrounding the TF (Fuller and Vermant, 2012). In most instances, liquid drains slower if these A-W interfaces possess more rheological stress as this would imply that they are more resistant to deformations (Hermans, Bhamla Saad, Kao, Fuller and Vermant, 2015). Proteins when adsorbed do so by developing a strongly viscoelastic film thereat (Murray, 2007). In contrast, lipids form a weakly viscoelastic monolayer at the A-W interface. When their interfacial concentration is locally reduced by surface convection, e.g. when the A-W interfaces surrounding the TF are deformed into a dimple (see above and Fig $\mathbf{1 A}$ ), lipids are inclined to restore this spatial concentration and thus $\sigma$ gradient. As such, they generate counteracting Marangoni stresses which decrease the rate of surface convection (Manikantan and Squires, 2020). Lipid molecules insoluble in the bulk phase do so by diffusing to regions of lower surface concentration and thus higher $\sigma$. Lipids soluble in the bulk phase can generate Marangoni stresses which depend on the rate of adsorption/desorption of these lipids at the A-W interface (Manikantan and Squires, 2020). Mixed protein-lipid interfaces generally have less rheological stress than A-W interfaces stabilized by proteins or lipids alone as proteins hinder lipids from generating Marangoni stresses while lipids prevent the formation of a coherent viscoelastic protein layer (Wilde, 2000). 

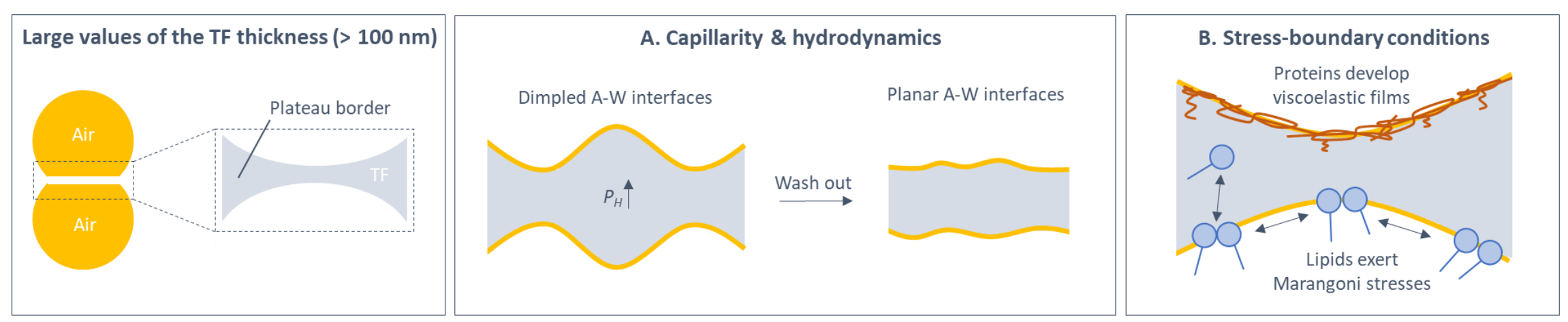

Drainage
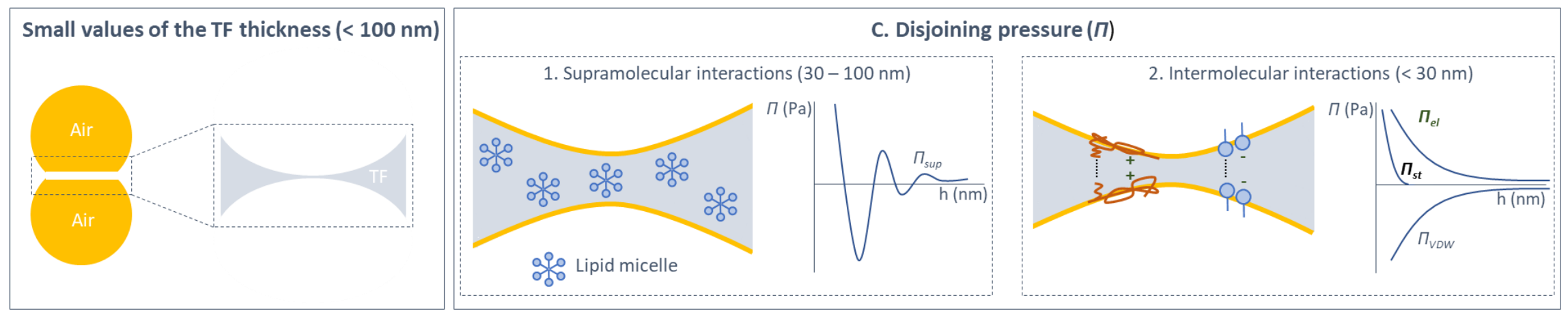

Fig 1. Graphical illustration of the interfacial and bulk phenomena determining the rate at which liquid drains from a thin film (TF) under a capillary hydrostatic

pressure $\left(P_{c}\right)$. At TF thickness values exceeding $100 \mathrm{~nm}$, the rate of liquid drainage largely depends on the shape (A) and the nature of the stress-boundary 
3. magnitude of $\boldsymbol{P}_{\boldsymbol{c}}$ (Eq 1), which is influenced by the pressure in the surrounding gas phase $\left(P_{g}\right)$ and the Laplace pressure due to the curvature in the Plateau border region of the TF. In turn, both $P_{g}$ and this Laplace pressure contribute to $P_{H}$ (Valkovska, Danov and Ivanov, 2002).

4. $\eta_{b}$, according to the Stefan-Reynolds equation $(1874 ; 1886)$ :

$$
\frac{d h}{d t}=\frac{-2 h^{3}}{3 \eta_{b} R_{T F}^{2}}\left(P_{C}-\Pi\right)(\text { Eq 1) }
$$

where $t$ is the drainage time, $h$ the TF thickness at time $t$, and $R_{T F}$ the TF radius. TFs prepared from high $\eta_{b}$ solutions drain slower than TFs prepared from low $\eta_{b}$ DL solutions. For DL TFs, $\eta_{b}$ is mostly determined by the concentration and structure of NSPs (Janssen et al. 2020a). Eq 1 is only valid for planar films of constant radius, and for the case of a no slip boundary condition. Although more elaborate models are required when the films become deformed (Dagastine et al. 2006; Vakarelski et al. 2010), the Stefan-Reynolds equation identifies the main factors related to film hydrodynamics, i.e. the thickness decrease is driven by the pressure drop and slowed down by the bulk viscosity.

At small TF thickness $(<100 \mathrm{~nm})$, the rate of liquid drainage strongly depends on the magnitude of $\Pi$. In turn, the contribution of $\Pi$ is function of the TF thickness and originates from supramolecular $\left(\Pi_{\text {sup }}\right)$ (Fig C1) (Nikolov and Wasan, 1989; Bergeron, Jimenez-Laguna and Radke, 1992; Bergeron and Radke, 1992) and intermolecular (Fig C2) (Derjaguin and Landau, 1941; Verwey and Overbeek, 1948) interactions between adsorbed constituents at both A-W interfaces. The former come into play when the TF is made up of one or more layers of supramolecular structures such as micelles (Nikolov and Wasan, 1989; Bergeron et al. 1992), or (synthetic) nanoparticles (Sethumadhavan, Nikolov and Wasan, 2001). The latter include attractive Van der Waals forces $\left(\Pi_{V D w}\right)$, repulsive electrostatic forces $\left(\Pi_{e l}\right)$, and repulsive steric forces $\left(\Pi_{s t}\right)$ (Damodaran, 2005). To fully understand gas cell stabilization in bread dough, insight in the drainage of liquid films between such gas cells would be of great interest. Against this background, for the first time, the drainage of wheat, rye, and oat DL TFs were assessed by interferometry using a bike-wheel shaped microcell similar to that of Cascão Pereira, Johansson, Blanch and Radke (2001), with modifications 
enabling assessing drainage dynamics over a wide range of driving pressures (Chatzigiannakis et al. 2020). TF measurements were performed with DLs (i) at bulk concentrations at which they occur in the respective supernatants after ultracentrifugation ('native concentration') and (ii) at lowered bulk concentrations. While the former measurements are the more relevant ones in the context of bread making, the latter allow evaluating the extent to which the outcome aligns with literature reports dealing with $A-W$ interfacial properties of diluted DL samples.

\section{Materials \& Methods}

191

\subsection{Materials}

Crousti bread wheat flour was purchased from Dossche Mills (Deinze, Belgium). Rye kernels (cultivar Dukato) from AVEVE (Merksem, Belgium) were conditioned to $16.0 \%$ moisture and subsequently roller milled in-house using a Bühler (Uzwil, Switzerland) MLU-202 laboratory mill as in Delcour, Vanhamel and De Geest (1989). Commercial oat flour was from Raisio Nutrition (Raisio, Finland).

Sugar, salt, and fresh compressed yeast (AB Mauri, Dordrecht, Nederland) were bought in a local supermarket. All chemicals, solvents, and reagents were at least of analytical grade.

A xylanase preparation (from Aspergillus aculeatus, Shearzyme 500L, containing 30,000 xylanase units $/ \mathrm{mL}$ ) was from Novozymes (Bagsvaerd, Denmark). Xylanase from A. aculeatus preferentially hydrolyzes the water-extractable AX population of wheat flour (Courtin and Delcour, 2001). A similar substrate specificity has been observed with rye flour water-extractable $A X$ (unpublished results). Lichenase (from Bacillus subtilis, containing 1,000 lichenase units $/ \mathrm{mL}$ ) and $\beta$-DGlucosidase (from Aspergillus niger, containing $40 \beta$-D-glucosidase units $/ \mathrm{mL}$ ) preparations were from the " $\beta$-D-glucan assay" kit of Megazyme (Bray, Ireland). Lichenases convert $\beta$-D-glucan into $\beta$-D-glucooligosaccharides by catalyzing the hydrolysis of $\beta-1,4$ bonds. $\beta$-D-glucosidases then selectively degrade 
these $\beta$-D-gluco-oligosaccharides into D-glucose molecules by catalyzing the hydrolysis of both $\beta-1,3$ and $\beta-1,4$ bonds (McCleary and Nurthen, 1986).

\subsection{Methods}

\subsubsection{Dough making and dough liquor isolation}

Wheat dough optimum water level and mixing time were determined using AACCI Methods 54-21.02 and 54-40.02, respectively. The bread making procedure was essentially that described by Finney (1984). Wheat flour (100.0 g), deionized water (54.73 mL), sugar (6.0 g), yeast (5.3 g), and salt (1.5 g) were mixed in a pin mixer (National Manufacturing, Lincoln, NE, USA) during $210 \mathrm{~s}$. In the case of rye and oat breads, the optimum water level was selected based on preliminary baking trials in which the water level resulting in the highest specific bread loaf volume was considered as optimal. In this case, the protocol in essence was that described by Hager, Bosmans and Delcour (2014). Rye or oat flour (265.0 g or $236.0 \mathrm{~g}$, respectively), deionized water ( $200.9 \mathrm{~mL}$ or $232.3 \mathrm{~mL}$, respectively), sugar (16.0 g or $14.8 \mathrm{~g}$, respectively), yeast (14.1 g or $13.1 \mathrm{~g}$, respectively), and salt (4.0 g or $3.7 \mathrm{~g}$, respectively) were mixed in a KitchenAid (St. Joseph, MI, USA) KPM5 mixer (60 s at speed 1 and $120 \mathrm{~s}$ at speed 4). All doughs/batters were fermented in a National Manufacturing fermentation cabinet $\left(30.0^{\circ} \mathrm{C}, 90 \%\right.$ relative humidity, $\left.126 \mathrm{~min}\right)$. Wheat doughs were sheeted in a National Manufacturing sheeter thrice during fermentation (roll gaps $4.8 \mathrm{~mm}$ after 52 and $77 \mathrm{~min}$ and $7.9 \mathrm{~mm}$ after $90 \mathrm{~min}$ ). Hydrating and mixing rye or oat flour results in poorly extensible dough and a cake-like batter, respectively. Therefore, rye doughs and oat batters could not be sheeted. While Janssen et al. (2018; 2020a;2020c) referred to the supernatant recovered from oat batter by ultracentrifugation as 'oat DL', we here more correctly refer to it as 'oat batter liquor' (oat BL). Fermented dough samples (ca. 15.0 $\mathrm{g}$, accurately weighed) were centrifuged at $165,000 \mathrm{~g}\left(20^{\circ} \mathrm{C}, 60 \mathrm{~min}\right)$ in $38 \mathrm{~mL}$ thick polycarbonate tubes (Beckman Coulter, Brea, CA, USA) with an L7 Ultracentrifuge (Beckman Coulter) and thereafter immediately placed on ice to minimize potential endogenous enzymatic activity. The supernatants 
(the DLs/BL) were collected in tared amber-colored Falcon tubes, weighed, flash frozen with liquid nitrogen, freeze-dried, and stored at $-18{ }^{\circ} \mathrm{C}$ until analysis. In previous work (Janssen, 2020b) it was established that freeze-drying does not alter the A-W interfacial properties of DL/BL constituents. These freeze-dried wheat and rye $\mathrm{DL}$ and oat $\mathrm{BL}$ powders were used for composition analysis (see section 2.2.2) and sample preparation (see section 2.2.3) prior to TF drainage measurements (see section 2.2.4).

\subsubsection{Dough/batter liquor chemical composition}

DL/BL protein $(\mathrm{N} \times 6.25)$ content was determined with an adaptation of AOAC method 990.03 to an automated 1108 Elemental Analyser (Carlo Erba, Hindley Green, UK) for microgram scale samples. DL/BL lipid levels were determined gravimetrically as in Janssen et al. (2020a; 2020c), based on the extraction protocol of Bligh and Dyer (1959). AX levels were determined as in Janssen et al. (2020a; 2020c) with a gas chromatography procedure in which flour samples were sequentially subjected to acid hydrolysis, reduction of monosaccharides to alditols, and subsequent conversion to alditol peracetates (Englyst and Cummings, 1984). $\beta$-D-Glucan levels were colorimetrically measured using a Megazyme (Bray, Ireland) protocol, which is also available as AACCI Method 32.23-01 (McCleary and Nurthen, 1986) as in Janssen et al. (2020a; 2020c). Protein, lipid, AX, and $\beta$-D-glucan levels were determined in duplicate on DLs/BLs from each of two separate isolations.

\subsubsection{Sample preparation}

TF measurements (see section 2.2.4) were carried out on wheat and rye DLs at 1.50 and 0.50 $\mathrm{mg}$ protein $/ \mathrm{mL}$, respectively, and oat $\mathrm{BL}$ at $1.00 \mathrm{mg}$ protein $/ \mathrm{mL}$, as well as on wheat $\mathrm{DL}$ and oat $\mathrm{BL}$ at their native concentrations (40.1 and $7.0 \mathrm{mg}$ protein $/ \mathrm{mL}$, respectively). In all cases, the most abundant NSP population (AX in wheat and rye DLs and $\beta$-D-glucan in oat $B L$ ) was depolymerized by enzyme(s) prior to performing TF measurements. Enzymatic treatments of wheat and rye DLs and oat BL were as follows. Freeze-dried wheat and rye DL powders were dissolved in deionized water (continuous 
stirring, $30 \mathrm{~min}, 23 \pm 2{ }^{\circ} \mathrm{C}$ ) at concentrations of 1.50 and $0.50 \mathrm{mg}$ protein $/ \mathrm{mL}$, respectively. Xylanase solution [containing $1.0 \mathrm{~mL}$ of xylanase preparation (see section 2.1) in $100 \mathrm{~mL}$ water] was added under gentle stirring to obtain a ratio of xylanase solution/DL dm protein of $2 / 1(\mathrm{v} / \mathrm{w})$. Freeze-dried oat $\mathrm{BL}$ powder was dissolved in deionized water (continuous stirring, $30 \mathrm{~min}, 23 \pm 2{ }^{\circ} \mathrm{C}$ ) at a concentration of $1.00 \mathrm{mg}$ protein $/ \mathrm{mL}$. Then, a solution of both lichenase and $\beta$-D-glucosidase [containing $0.50 \mathrm{~mL}$ of both the lichenase and $\beta$-D-glucosidase preparations (see section 2.1 ) in 100 $\mathrm{mL}$ water] was added under gentle stirring to obtain ratios of enzyme solution/DL $\mathrm{dm}$ protein of $1 / 1$ $(v / w)$ for each of the enzymes present. The respective enzymatic treatment of wheat $D L$ and oat $B L$ at their native concentrations was as above with the exception that the ratio of total enzyme solution/DL or $\mathrm{BL} d \mathrm{~m}$ protein was $10 / 1(\mathrm{v} / \mathrm{w})$ instead of $2 / 1(\mathrm{v} / \mathrm{w})$. The sample volume required for TF measurements was approximately $5.0 \mathrm{~mL}$.

\subsubsection{Analysis of dough/batter liquor thin film drainage dynamics}

TF measurements were carried out on the wheat and rye $\mathrm{DL}$ and oat $\mathrm{BL}$ samples described in section 2.2.3. Fig 2 shows the TF balance set-up used. The design of the bike-wheel shaped microchip was based on that of Cascão Perreira et al. (2001) and identical to that of Beltramo and Vermant (2016). More recently, Chatzigiannakis et al. (2020) modified the TF set-up allowing assessing drainage dynamics by reducing compliance of the setup and adding adequate pressure control systems. It is this modified TF set-up that was used in the present study. The microchips were made on borosilicate glass by photolithography (Micronit Microfluidics, Enschede, The Netherlands) and consisted of a diamond bit drilled central orifice (diameter: $1 \mathrm{~mm}$, thickness: $400 \mu \mathrm{m}$ ) connected to a larger circular channel (width: $500 \mu \mathrm{m}$, depth: $50 \mu \mathrm{m}$ ) via 25 smaller channels (width: $45 \mu \mathrm{m}$, depth: $20 \mu \mathrm{m}$, length: $800 \mu \mathrm{m}$ ) oriented in a radial pattern. The chip was glued to the top of a titanium T-shaped holder using two component epoxy adhesives (Fig 2A). Before use, the walls of the bike-wheel chip were rendered hydrophilic by immersion and ultra-sonication in sodium hydroxide saturated ethanol. After creating a DL/BL thick film in the central orifice by means of a $1.0 \mathrm{~mL}$ syringe coupled to rigid 

was then thoroughly sealed to avoid leaks and connected to a piezoelectric pressure control system control system has a resolution of $1 \mathrm{~Pa}$ and a response time of $10 \mathrm{~ms}$.

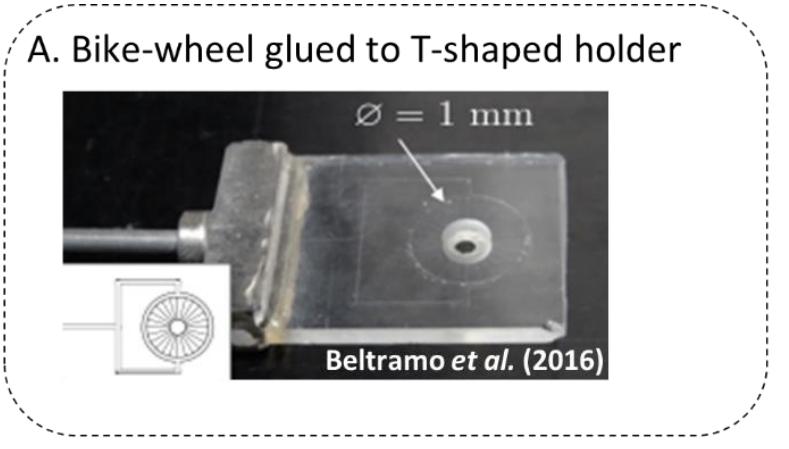

\section{B. Pressure control system and chamber}

C. Side view of DL TF in bike-wheel
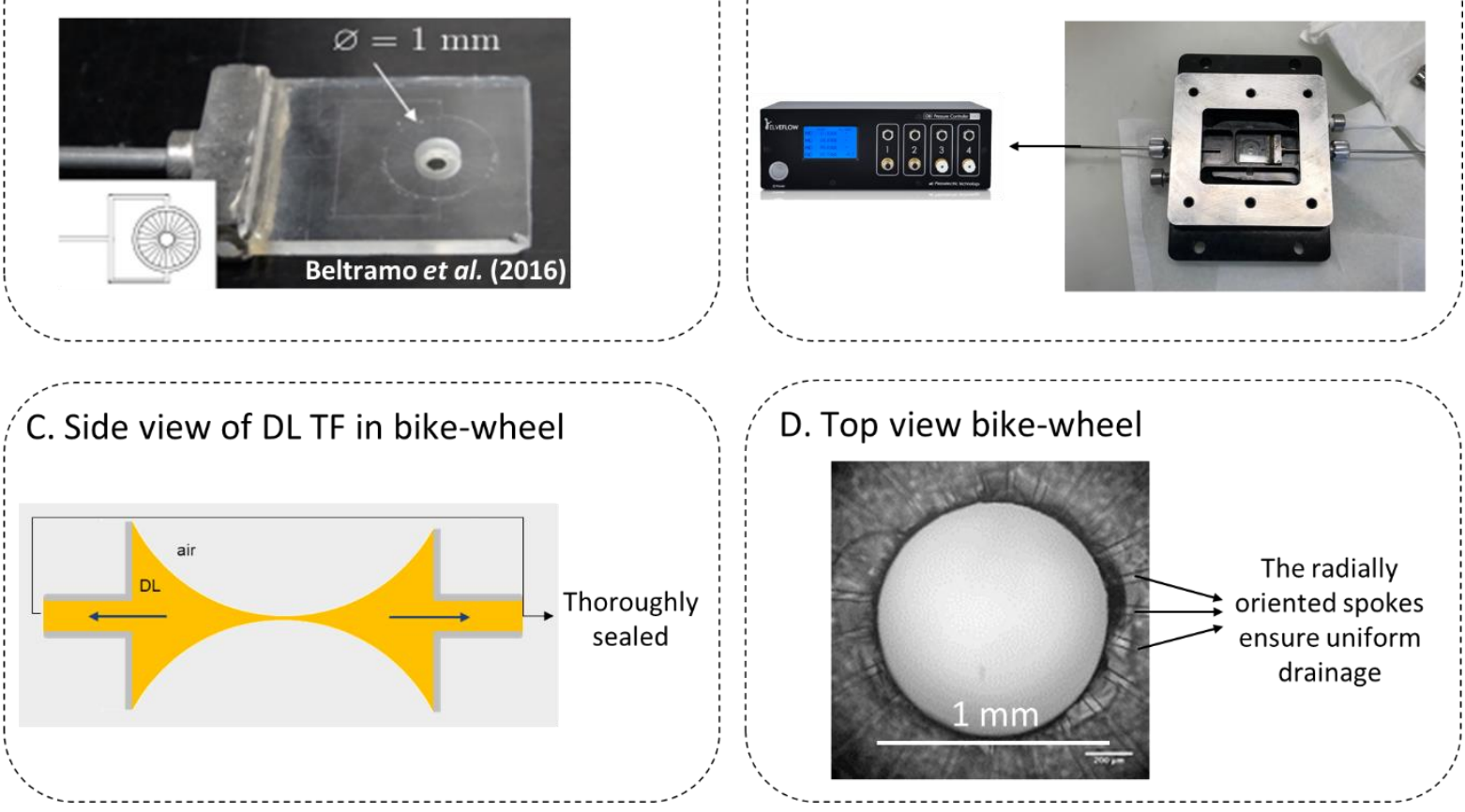

Fig 2. Thin film (TF) interferometry set-up. A. Photograph of the hydrophilic bike-wheel glued to the T-

shaped holder (Chatzigiannakis et al. 2020). B. Aluminum pressure chamber in which the bike-wheel is

mounted. This chamber is connected to a piezoelectric pressure control system. C. Schematic side view of a dough liquor (DL) TF (in yellow) in the inner orifice of the bike-wheel. The end of the T-shaped holder to which the bike-wheel is glued is thoroughly sealed. D. Top view of the inner orifice of the bikewheel. The radially oriented channels allow uniform drainage from the central orifice.

TF experiments were executed as follows. First, the TF was carefully drained by increasing $P_{g}$ in small discrete steps until the first interference fringes appeared and the film remained stable, i.e. the TF neither thickened nor thinned. At this point, the contribution of $\Pi$ to the total pressure in the TF (the difference between $P_{c}$ and $\Pi$ ) is assumed to be negligible when the TF had a thickness $>100$ 
$\mathrm{nm}$. Thus, the first interference fringes remained stable because $P_{c}$ was zero (Bergeron and Radke, 1992):

$$
\begin{gathered}
P_{C}=P_{g}+P_{L, b w}=0(\text { Eq 2) } \\
P_{C}=P_{g}+\frac{2 \sigma}{R_{b w}}=0(\text { Eq 3) }
\end{gathered}
$$

where $P_{L, b w}$ is the Laplace pressure due to the natural curvature of the film in the bike-wheel and where $R_{b w}$ is the radius of the bike-wheel's hole $(500 \mu \mathrm{m})$. Subsequently, drainage of the TF was forced by applying an excess $P_{g}$ of either $50 \mathrm{~Pa}\left(e P_{g}{ }^{50 P a}\right)$ or $200 \mathrm{~Pa}\left(e P_{g}{ }^{200 P a}\right)$. From this point onwards, the TF reduced its thicknesses and there was a contribution of $\Pi$ to the total pressure in the TF (the difference between $P_{c}$ and $\Pi$, Eq 1). In addition, there was a contribution of hydrodynamic pressures $\left(P_{H}\right)$ to $P_{c}$ because liquid flow in the TF took place. Thus, liquid drains from the TF when:

$$
P_{c}>\Pi(\text { Eq 4), and thus when }
$$

$$
P_{g}+e P_{g}+\frac{2 \sigma}{R_{b w}}-P_{H}>\Pi(\text { Eq 5) }
$$

From a certain TF thickness, the radius of the TF increases until it either ruptures or over time reaches an equilibrium radius $\left(R_{T F, e q}\right)$. The latter is defined by the pressure balance at the Plateau border region of the TF (Toshev and Ivanov, 1975):

$$
R_{T F, e q}^{2}=R_{b w}^{2}-\left(\frac{2 \sigma R_{b w}}{e P_{g}+P_{L, b w}}\right)(\mathrm{Eq} 6)
$$

Thus, in case no rupture takes place, a TF subjected to a larger $e P_{g}$ expands to a larger $R_{T F, e q}$ than when a lower $e P_{g}$ is applied.

In case no TF rupture took place in the time frame of the experiment, the bike-wheel was thoroughly rinsed with water and filled with new DL/BL material prior to initiating a new measurement. When it did rupture, a new thick film was formed by lowering the $e P_{g}$ to $0 \mathrm{~Pa}$ and the measurement was repeated as outlined above. The latter was possible because the end of the holder to which the bike-wheel chip was glued was thoroughly sealed (Fig $\mathbf{2 C}$ ). The total time required for preparing the sample and reaching the point at which $P_{c}$ was zero (Eq 3 ) was approximately $8 \mathrm{~min}$ for all $\mathrm{DL} / \mathrm{BL}$ samples. Thus, A-W interface aging was limited before drainage was forcibly induced. All 
repetitions for a given $\mathrm{DL} / \mathrm{BL}$ sample were executed within 30 min to prevent aging of the bulk phase.

Table S1 (supplementary information) provides an overview of the total number of drainage experiments performed for the different samples.

Throughout the experiments, TFs were viewed with an upright microscope (Nikon Eclipse FN1, Nikon Instruments, Melville, USA) equipped with a 10x long working distance objective (numerical aperture of 0.30) and a camera (ORCA-flash4.0 CMOS, Hamamatsu Photonics, Herrsching, Germany) (Fig 2D). Images were stored in .tiff format (2048 x 2048 pixels) with a maximal temporal resolution of $10 \mathrm{~ms}$ and further processed using ImageJ software (National Institute of Health, Bethesda, MD, USA).

A drainage time could only be determined in case of TF rupture and was defined as the time between the onset of TF expansion and rupture. When the TF ruptured, TF expansion rates were calculated by subtracting the initial TF radius (about $25 \mu \mathrm{m}$ ) from the TF radius just before rupture and dividing the resulting value by the drainage time. When the TF did not rupture, TF expansion rates were calculated by subtracting the initial TF radius from $R_{T F, e q}$ and dividing the resulting value by the time needed to reach $R_{T F, e q}$. A TF is expected to expand at a slower rate when the surrounding A-W interfaces provides more resistance to an applied deformation. To better understand this relationship, Boussinesq (Bq) numbers were calculated as:

$$
B q=\frac{\left|\eta_{S}^{*}\right|}{\eta_{b} R_{T F, a v}}(\text { Eq 7) }
$$

where $\left|\eta_{s}\right|$ is the magnitude of the complex interfacial shear viscosity, taken here at a single frequency of $0.5 \mathrm{~Hz}$ and $8 \mathrm{~min}$ after the start of adsorption, and $R_{T F, a v}$ the average TF radius of the drainage experiment. The $\eta_{s}{ }_{s}$ and $\eta_{b}$ were determined with a standard rheometer equipped with a double wall ring (Vandebril, Franck, Fuller, Moldenaers and Vermant, 2010) and double wall Couette geometry, respectively, as in Janssen et al. (2020a). For oat BL TFs, $\left|\eta^{*}\right|$ was lower than the detection limit of the set-up (5.0 $\times 10^{-6}$ Pa.s.m), so this value was used for calculating Bq numbers as an upper limit. 
using the Sheludko (1967) equation:

$$
h_{e q}=\left(\frac{\lambda}{2 \pi n_{f}}\right)\left(m \pi \pm \arcsin \sqrt{\frac{\Delta}{1+4 Q \frac{(1-\Delta)}{(1-Q)^{2}}}}\right)(\text { Eq 8) }
$$

$$
\Delta=\frac{I_{f}-I_{\min }}{I_{\max }-I_{\min }}\left(\mathrm{Eq} \text { 9) } \quad Q=\left(\frac{n_{f}-n_{c}}{n_{f}+n_{c}}\right)^{2}(\mathrm{Eq} \text { 10) }\right.
$$

with $\lambda$ the wavelength of the monochromatic light used for reflection $(508 \mathrm{~nm}), n_{f}$ and $n_{c}$ the refractive indices of the $\operatorname{TF}(1.33$, the refractive index of water) and air (1.00028), respectively, $m$ the order of interference, $I_{f}$ the intensity or grey value of one pixel of a particular TF image, $I_{\max }$ and $I_{\min }$ the maximum and minimum intensities or grey values, respectively, of the pixels of the TF region of all images obtained for a given TF drainage experiment (Exerowa and Kruglyakov, 1997). As the refractive indices were not determined, $h_{\text {eq }}$ represents a calculated equivalent TF thickness of the pixel area analyzed. The TF $h_{e q}$ values reported throughout this manuscript represent average values of several such areas of at least three different TF images (Table S1). It should be noted that TF thicknesses obtained via the Sheludko equation must be interpreted with care as it assumes (i) a planar film, (ii) constant $I_{\max }$ and $I_{\min }$ during the drainage experiment, (iii) constant refractive TF index,

\section{Results and discussion}

\subsection{Dough liquor chemical composition}

In previous work by our group, the chemical composition of the wheat and rye DLs and oat BL used here has been discussed in-depth (Janssen et al. 2018; 2020a; 2020b; 2020c). In short, lipid levels were high in oat $\mathrm{BL}(29.7 \%$ of $\mathrm{BL} \mathrm{dm})$ and much lower in rye $(1.5 \%$ of $\mathrm{DL} \mathrm{dm})$ and wheat $(0.5 \%$ of $\mathrm{DL}$ $\mathrm{dm})$ DLs. The protein levels decreased in the order wheat ( $16.9 \%$ of $\mathrm{DL} \mathrm{dm})$, rye ( $14.0 \%$ of $\mathrm{DL} \mathrm{dm})$, and 
respectively), while $\beta$-D-glucan was the most abundant NSP in oat BL (11.9\% of BL dm). The aqueous phase of cereal doughs apart from proteins, lipids, and NSPs, contains mono-, di- and oligosaccharides (Primo-Martín et al. 2006; Salt et al. 2006) and probably also minor levels of minerals and vitamins (Belitz, Grosch and Schieberle, 2009).

\subsection{Dough/batter liquor thin film drainage dynamics}

TF drainage measurements were conducted both after applying $e P_{g}^{50 P a}$ and $e P_{g}{ }^{200 P a}$, which allowed mimicking the drainage of TFs separated by gas cells of different sizes. As a point of reference, the average radius of gas cells in dough increases from about $100 \mu \mathrm{m}$ up to $1000 \mu \mathrm{m}$ over the course of fermentation (Babin et al. 2006; Turbin-Orger et al. 2015a). Thus, according to the Young-Laplace equation:

$$
P_{L, \text { gas cell }}=\sigma\left(\frac{1}{R_{1}}+\frac{1}{R_{2}}\right)(\text { Eq 11) }
$$

where $P_{L, \text { gas cell }}$ is the pressure difference across the gas cell A-W interface and $R_{1}$ and $R_{2}$ the gas cell radii in the $\mathrm{x}$ and $\mathrm{y}$ directions, respectively, perfectly spherical gas cells (for which $R_{1}$ equals $R_{2}$ ) with an average $\sigma$ of $40 \mathrm{mN} / \mathrm{m}$ experience $P_{L \text {, gas cell }}$ values between 800 and 80 Pa during fermentation. As the gluten network surrounding gas cells presumably only ruptures during the late stages of fermentation, it seems reasonable to assume that gas cells regionally surrounded by a TF only indeed experience $P_{L, \text { gas cell }}$ values in the range of those applied in the present experiments. As the phenomena observed after applying $e P_{g}{ }^{50 P a}$ and $e P_{g}{ }^{200 P a}$ were very similar, the results obtained for $e P_{g}{ }^{200 P a}$ are discussed in-depth, while interferometry images obtained at $e P_{g}{ }^{50 P a}$ are provided as supplementary data.

First, the drainage dynamics of wheat and rye DL TFs at 1.50 and $0.50 \mathrm{mg}$ protein $/ \mathrm{mL}$, respectively, and oat $B L \mathrm{TFs}$ at $1.00 \mathrm{mg}$ protein $/ \mathrm{mL}$ were analyzed. Although these concentrations were well below those in the respective supernatants after ultracentrifugation, these measurements allow rationalizing the findings reported in literature thus far (see section 1). Second, the drainage of wheat $\mathrm{DL}$ and oat $\mathrm{BL}$ TFs at their native concentrations was monitored to assess the potential of 
aqueous phase constituents as they occur in dough to stabilize gas cells during bread making. Videos illustrating the drainage processes of all above-mentioned TFs, both after applying $e P_{g}{ }^{50 P a}$ and $e P_{g}{ }^{200 P a}$, are included as supplementary information. Note that in case of the videos of TFs at native concentration one frame was selected every $9 \mathrm{~s}$.

Some limitations of the experimental set-up used must be considered. First, rye DL TFs at their native concentration could not be monitored because rye DL constituents formed large aggregates in the microchip channels which prevented drainage to occur freely. Second, the TF balance set-up used was limited to liquids with sufficiently low $\eta_{b}$. Here, $\eta_{b}$ was drastically reduced (Janssen et al. 2020a) by enzymatically depolymerizing the respective NSP populations (see section 2.2.3) prior to performing TF measurements. As mentioned earlier, $\eta_{b}$ would directly affect TF drainage (Eq 1). In earlier work, $\eta_{b}$ of the DLs/BL used in the present study have been reported on, which allows estimating their likely impact on TF drainage (Janssen et al. 2020a).

\subsubsection{Drainage dynamics of wheat and rye dough liquor and oat}

\section{batter liquor thin films at lowered bulk concentrations}

\subsubsection{Thin film structural changes}

\section{WHEAT DOUGH LIQUOR}

Fig 3 shows interferometry images of wheat DL TFs at $1.50 \mathrm{mg}$ protein $/ \mathrm{mL}$ at different time points after applying $e P_{g}^{200 P a}(\mathbf{A}$ to $\mathbf{D})$. The 3D intensity plot of the image just before rupture in Fig 3D is shown in Fig 3E. Just before rupture, at $\mathrm{e}_{g}{ }^{200 P a}$, wheat DL TFs at $1.50 \mathrm{mg}$ protein $/ \mathrm{mL}$ were characterized by white regions near the Plateau border, whereas their central part consisted of white spots dispersed in a dark grey region (Fig 3D). In these measurements, such white and dark grey regions generally represent the thickest and thinnest part of the TF, respectively. The 3D pixel intensity plot of the image in Fig 3D (Fig 3E) further illustrates this. This thickness assumption is only valid when comparing TF regions with $h_{e q}$ values in the same order of interference. For instance, the $h_{e q}$ values of 
the black regions in Figs $\mathbf{3 B}$ and $\mathbf{3 C}$ have a greater thickness than the white regions in Fig $\mathbf{3 C}$ or dark grey regions in Fig 3D because the order of interference is higher in the former. Just before rupture (Fig 3D), the white regions near the plateau border and the dark grey regions in the TF had average $h_{e q}$ values of $76 \pm 5 \mathrm{~nm}$ and $20 \pm 1 \mathrm{~nm}$, respectively. The white regions likely consist of a relatively thick film surrounded by two A-W interfaces. In contrast, it is reasonable to assume that $h_{e q}$ of the dark grey region represents the combined thicknesses of both $\mathrm{A}-\mathrm{W}$ interfaces, as one can expect that at this point just before rupture little if any liquid remains between them. It was demonstrated in earlier work that at similar lowered bulk concentrations, both proteins and lipids are present at wheat DL stabilized A-W interfaces (Janssen et al. 2020c). Using interferometry and ellipsometry, the thicknesses of $\beta$-casein and bovine serum albumin TFs just before rupture have been estimated to be $17-26 \mathrm{~nm}$ and $4-12 \mathrm{~nm}$, respectively, depending on the $\mathrm{pH}$ and ionic strength of the bulk phase, the aging time of the $\mathrm{A}-\mathrm{W}$ interface, and the magnitude of $e P_{g}$ (Cascão Pereira et al. 2003). Furthermore, $h_{e q}$ of an inverse lipid bilayer or common black film corresponds to the sum of (i) twice the characteristic length of the hydrocarbon chains of the participating lipid molecules and (ii) the thickness of the aqueous layer separating both lipid monolayers. Common black films typically have $h_{\text {eq }}$ values in the range of $10-20 \mathrm{~nm}$, although the exact value depends on the contributions of $\Pi_{V D W}$ and $\Pi_{e l}$ (Zhang, Yilixiati, Pearsall and Sharma, 2016). Based on the above for pure protein and lipid films, it thus seems plausible that the dark grey region in Fig 3D, which was about $20 \mathrm{~nm}$ thick, represents two opposing mixed protein-lipid A-W interfacial films, as was suggested in earlier work to be the case based on interfacial rheology measurements (Janssen et al. 2020c). The white spots dispersed in the central grey region had $h_{e q}$ values between 60 and $70 \mathrm{~nm}$ (Fig 3D) and appeared as discrete entities in the 3D pixel intensity plot (Fig 3E). Such entities have previously been observed in 440 TFs prepared from isolated proteins and have been interpreted to represent protein aggregates 441 (Maldonado-Valderrama and Langevin, 2008; Rullier et al. 2009; Rullier et al. 2010; Gochev et al. 2014; 442 Chen et al. 2018). 


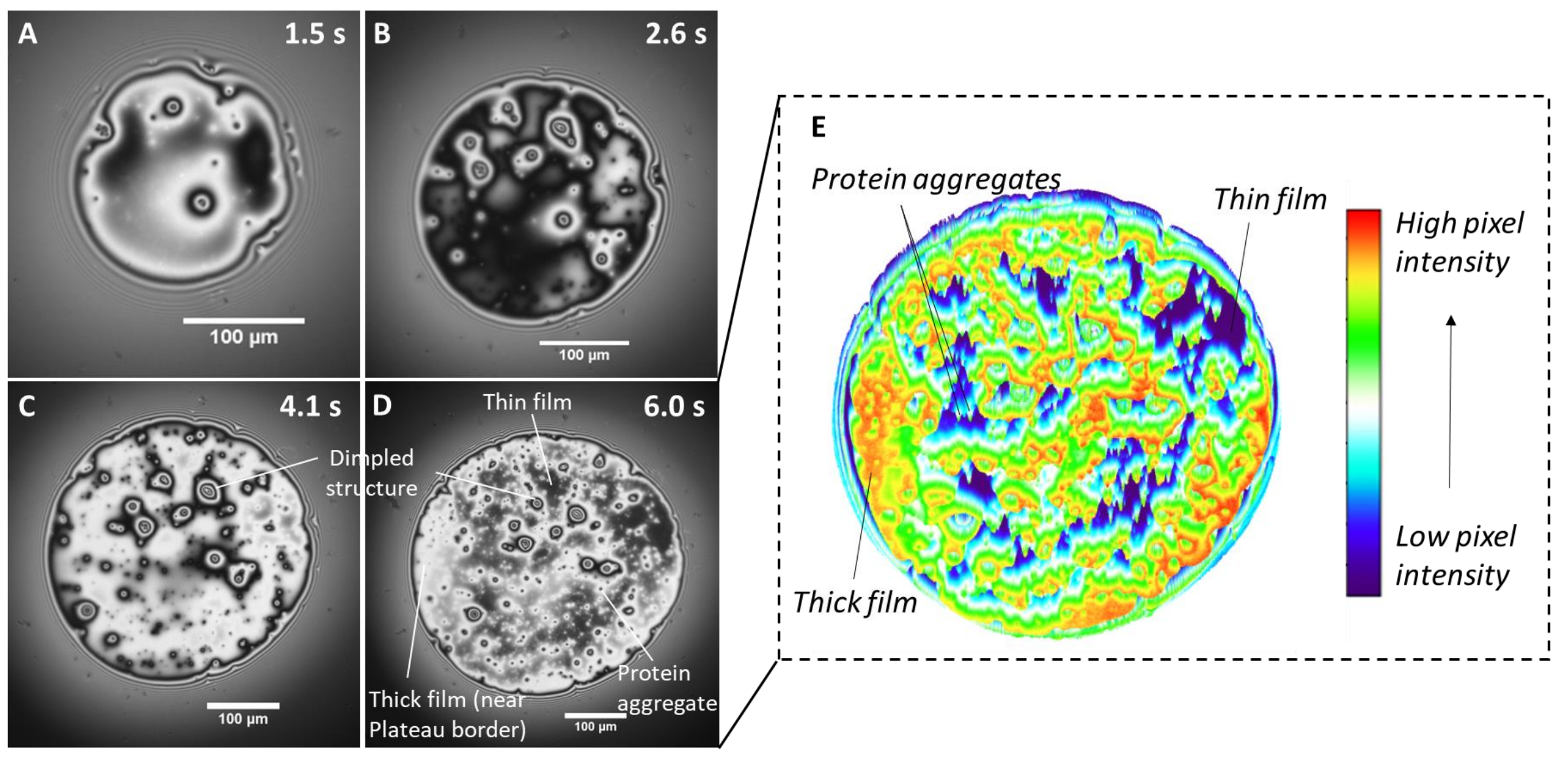

Fig 3. Interferometry images of wheat dough liquor (DL) thin films (TFs) at $1.50 \mathrm{mg}$ protein/mL at different time points after applying an excess gas pressure of $200 \mathrm{~Pa}\left(\mathrm{eP}_{\mathrm{g}}{ }^{200 \mathrm{~Pa}}\right)$. D represents the center part of the image just before TF rupture and is presented in $E$ as a 3D intensity plot, in which lower TF pixel 

rings were observed (Figs 3A to 3D). Each ring corresponds to one order of reflected light interference or - in this case - an $h_{e q}$ equal to $\Lambda / 4 n$ or $95 \mathrm{~nm}$. Thus, the number of concentric rings provides a rough estimate of the thickness at the center of those spots. In Fig 3D, the $h_{e q}$ values at the center of the concentric ring structures varied between 145 and $320 \mathrm{~nm}$. Thus, these concentric ring structures represented the thickest parts of wheat DL TFs at $1.50 \mathrm{mg}$ protein/mL. Rullier et al. $(2009 ; 2010)$ monitored the drainage of TFs prepared from solutions containing $\beta$-lactoglobulin aggregates and observed such concentric ring structures. Similar observations for protein aggregates were also made in other studies (Cascão Pereira et al. 2003; Chen et al. 2017; Chen et al. 2018). All in all, the concentric ring structures in Figs 3A to 3D likely represent larger protein aggregates than those in the earlier mentioned white and black spots. Interestingly, protein aggregates at the A-W interface coalesced when liquid drainage progressed, presumably a result of compression due to film thinning. At $e P_{g}^{50 P a}$ (see supplementary information), smaller aggregates and no concentric ring structures were observed. This is probably related to less pronounced protein unfolding and subsequent aggregation at lower hydrostatic pressures (Dumay, Kalichevsky and Cheftel, 1994; Messens, Van Camp and Huyghebaert, 1997).

\section{RYE DOUGH LIQUOR}

Fig 4 shows interferometry images of rye DL TFs at $0.50 \mathrm{mg}$ protein $/ \mathrm{mL}$ at different time points after applying $e P_{g}{ }^{200 P a}(A$ to $\mathbf{D})$. It was not possible to create a 3D intensity plot for rye DL TFs at $e P_{g}{ }^{200 P a}$ because the A-W interfaces of these TFs were dimpled (Fig 1A) and would have resulted in distorted 3D intensity plots. In addition, smaller black spots and concentric ring structures were observed (Figs 4A to 4D), with estimated $h_{e q}$ values at their center ranging between 100 and $300 \mathrm{~nm}$. As discussed above for wheat DL TFs, these concentric ring structures in rye DL TFs at $0.50 \mathrm{mg}$ protein/mL (Figs 4A to 4D) most likely represent protein aggregates. Similar to what was observed for wheat DL TFs, protein aggregates at the A-W interfaces surrounding rye DL TFs coalesced during the drainage process, implying flow induced aggregation as the TF thinned. 
474 border (Figs 4C and 4D), with an average $h_{e q}$ value of $79 \pm 3 \mathrm{~nm}$. It has been shown that proteins are

475 the dominant constituents at rye DL stabilized A-W interfaces (Janssen et al. 2020c). However, the

476 average $h_{e q}$ of said white regions (79 $\mathrm{nm}$ at $e P_{g}{ }^{200 P a}$ ) was much higher than that of protein stabilized

477 A-W interfacial films [4 to $26 \mathrm{~nm}$ (Cascão Pereira et al. 2003)]. This indicates that these white regions
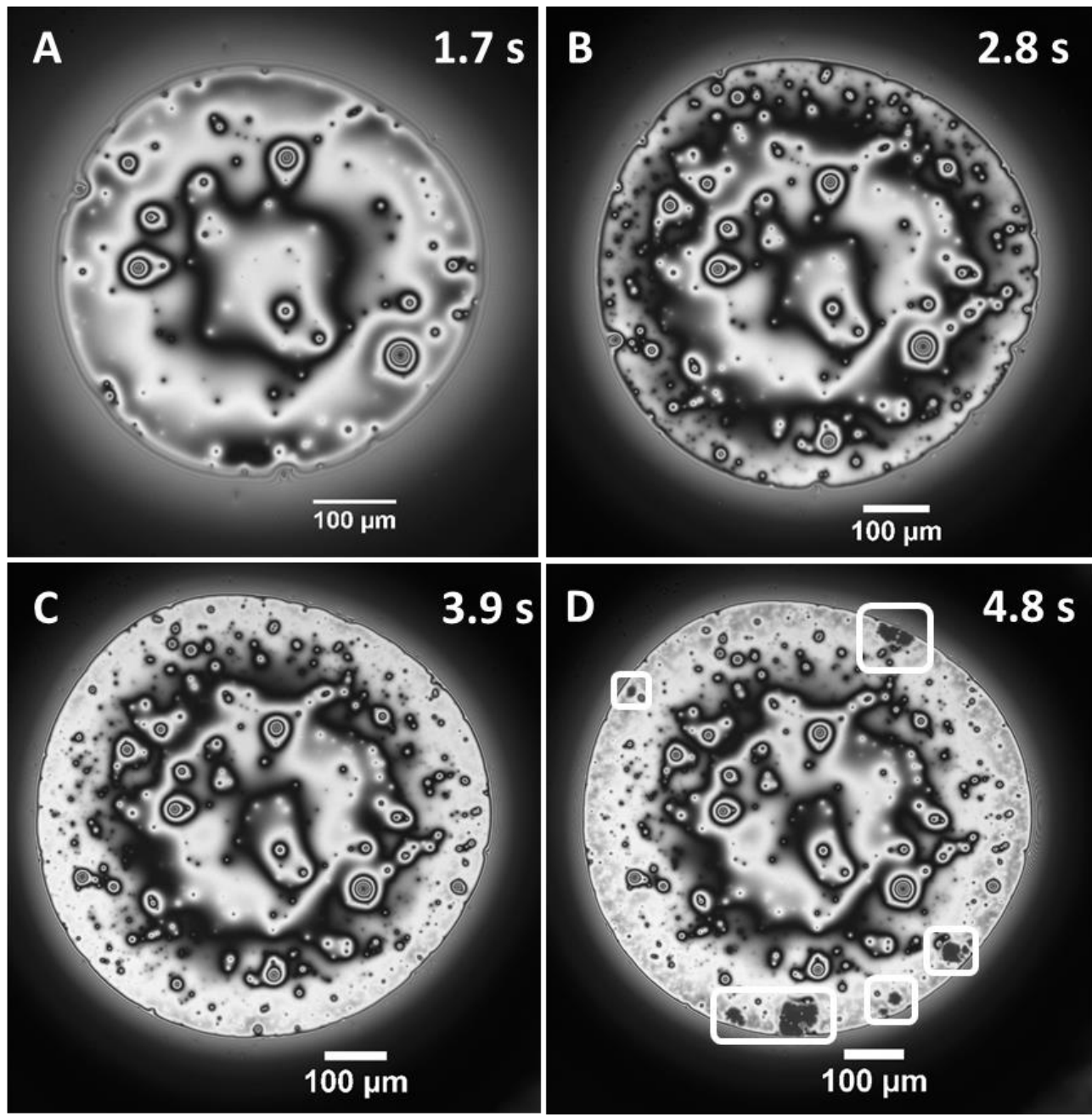

Fig 4. Interferometry images of rye dough liquor (DL) thin films (TFs) at $0.50 \mathrm{mg}$ protein/mL at different

time points after applying an excess gas pressure of $200 \mathrm{~Pa}\left(e_{\mathrm{P}}{ }^{200 \mathrm{~Pa}}\right)$. D represents the image just before TF rupture. The dark grey regions in the white rectangular boxes are the result of steric and/or electrostatic repulsive forces occurring between proteins at the two opposing air-water interfaces. 
(highlighted by white boxes in Fig 4D) and expanded over time. These regions had an $h_{e q}$ of $20 \pm 1 \mathrm{~nm}$, thus representing the part of the TF with the lowest thickness. Very similar observations were made at $e P_{g}{ }^{50 P a}$ (see supplementary information). As these spots appeared just before TF rupture, it can be assumed that merging of the A-W interfaces occurred in these regions. The onset and expansion of noncircular thin regions as observed in Fig 4D has earlier been described by Cascão Pereira et al. (2003) for $\beta$-casein and bovine serum albumin TFs and by Gochev et al. (2014) for $\beta$-lactoglobulin TFs. Establishing $\Pi$-area isotherms revealed that the occurrence of such regions is the result of both electrostatic and steric repulsive forces between proteins adsorbed at the A-W interfaces surrounding the TF (Cascão Pereira et al. 2003; Gochev et al. 2014). That proteins dominate rye DL stabilized A-W interfaces is in line with previous findings (Janssen et al. 2020a; Janssen et al. 2020c).

\section{OAT BATTER LIQUOR}

Fig 5 shows interferometry images of oat BL TFs at $1.00 \mathrm{mg}$ protein $/ \mathrm{mL}$ at different time points after applying $e P_{g}{ }^{200 P a}(A$ to $\mathbf{D}) .3 \mathrm{D}$ intensity plots could be made from oat BL TF interferometry images neither at $e P_{g}{ }^{50 P a}$ (see supplementary information) nor at $e P_{g}^{200 P a}$ because the TFs were pronouncedly dimpled. Indeed, at $e P_{g}{ }^{200 P a}$ a symmetric dimple was observed in oat $\mathrm{BL}$ TFs at $1.00 \mathrm{mg}$ protein $/ \mathrm{mL}$ (Figs 5A and 5B). It was visually observed that black and white spots migrated to the center of the dimple (Fig 5A). This indicates that the oat BL constituents in these spots were inclined to restore the $\sigma$ gradient which originated from dimple formation (see section 1). This implies that Marangoni effects, which are typically observed for low molecular weight surfactant-stabilized interfaces (Damodaran, 2005), were at play here. Lipids thus likely dominated the A-W interfaces in oat BL TFs at $1.00 \mathrm{mg}$ protein/mL. This agrees well with our earlier observations (Janssen et al. 2020c). 

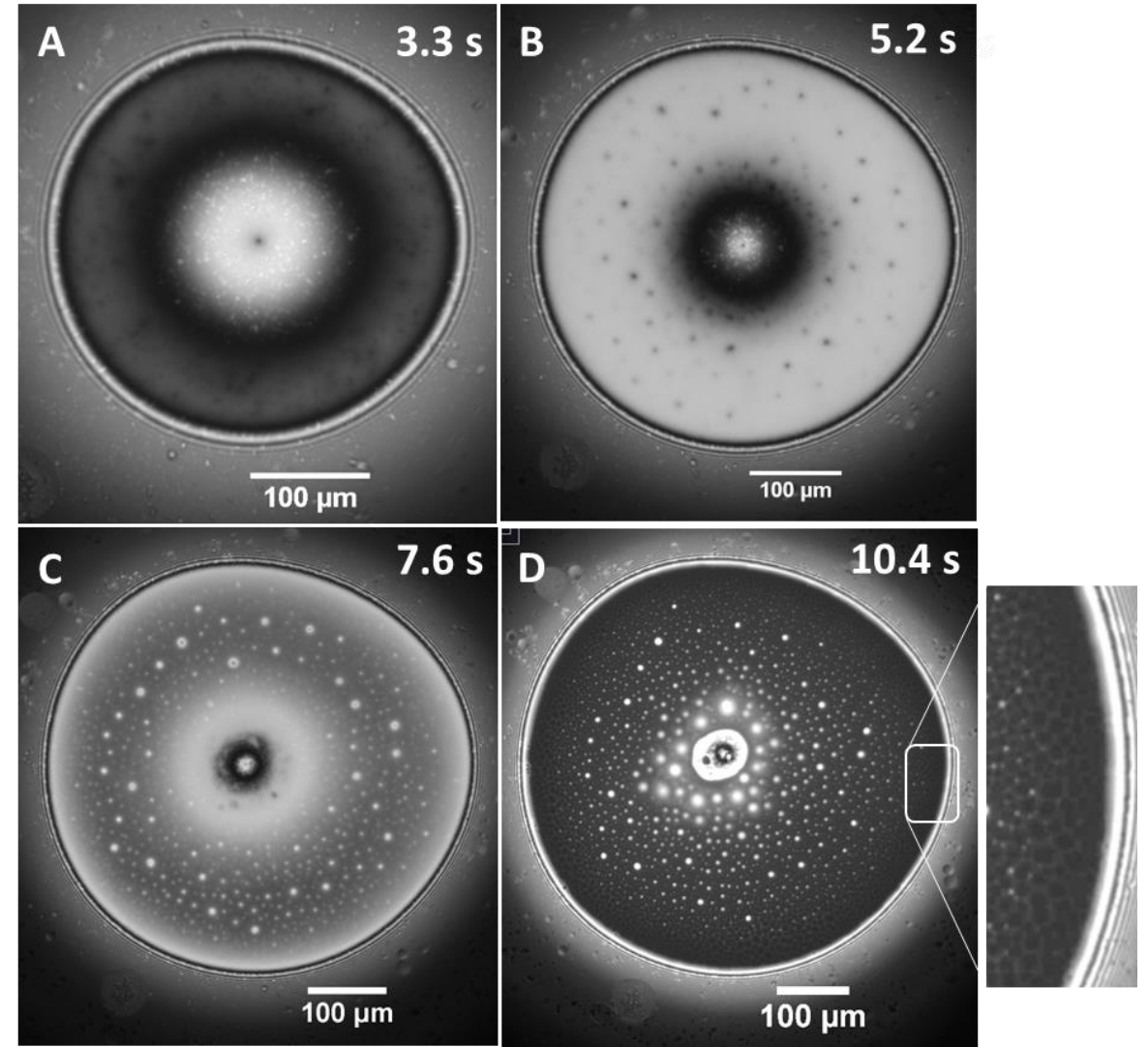

Fig 5. Interferometry images of oat batter liquor (BL) thin films (TFs) at $1.00 \mathrm{mg}$ protein/mL at different time points after applying an excess gas pressure of $200 \mathrm{~Pa}\left(e \mathrm{P}_{g}^{200 P a}\right)$. D represents the image just before TF rupture.

As drainage progressed, somewhat contradictory results were obtained in the various repetitions of the oat $\mathrm{BLTF}$ drainage experiments at $e P_{g}{ }^{200 P a}$. In a first set of experiments, TFs ruptured when the A-W interfaces were still dimpled, which implies that TFs ruptured near the Plateau border (the thinnest region of the dimple). However, in a second set of experiments, the dimple contracted progressively, indicating that the A-W interfaces evolved towards a planar shape over time. This was followed by the appearance of white spots dispersed in a black region (Figs $\mathbf{5 C}$ and $\mathbf{5 D}$ ). This black region had an average $h_{\text {eq }}$ of $12 \pm 1 \mathrm{~nm}$ just before rupture and most likely represents an inverse lipid bilayer or common black film. Interestingly, these black regions were embedded in a thicker, grey network-like structure (Fig 5D). As will be addressed in more detail in section 3.2.2.1, this implies that oat BL TFs at $1.00 \mathrm{mg}$ protein/mL display stratification due to the presence of one or multiple layers of micelles. The presence of micelles in oat BL TFs at $1.00 \mathrm{mg}$ protein $/ \mathrm{mL}$ is supported by the outcome 
of $\sigma$ measurements at increasing DL bulk concentration (see supplementary information), which indicated that the critical micelle concentration was reached at $0.10 \mathrm{mg}$ protein $/ \mathrm{mL}$. The abovementioned white spots were present as distinct circular entities and had average $h_{e q}$ values ranging between 30 and $60 \mathrm{~nm}$ (Fig 5D). As Marangoni stresses were observed during drainage of oat BL TFs at $1.00 \mathrm{mg}$ protein $/ \mathrm{mL}$ at $e P_{g}{ }^{200 P a}$ (Fig $\mathbf{5 A}$ ), these entities were probably not protein aggregates. Interestingly, most of these white spots were located at the rim of the black regions and seemed to be part of the grey network-like structure. Therefore, they may represent liquid expelled during the transition of a TF region in which one layer of micelles is enclosed (i.e. the grey network) to a TF region representing an inverse lipid bilayer or common black film (i.e. the black regions). A similar effect has been observed for sodium dodecyl sulfate (SDS) TFs (Bergeron et al. 1992; Zhang et al. 2016).

\subsubsection{Thin film stability}

Table 1 shows several parameters characteristic for the drainage experiments carried out with wheat and rye DL TFs at 1.50 and $0.50 \mathrm{mg}$ protein $/ \mathrm{mL}$, respectively, as well as of oat BL TFs at $1.00 \mathrm{mg}$ protein/mL. Each of the three TFs ruptured in less than fifteen seconds, both at $e P_{g}^{50 P a}$ and $e P_{g}{ }^{200 P a}$, indicating that they were unstable and that the A-W interfaces surrounding the TF merged rapidly (Table 1).

Despite the similar TF stabilities, some notable differences in the drainage dynamics of wheat and rye $\mathrm{DL}$ and oat BL TFs were observed. Wheat DL TFs at $1.50 \mathrm{mg}$ protein $/ \mathrm{mL}$ had lower expansion rates than rye $\mathrm{DL}$ and oat $\mathrm{BL} T F s$ at 0.50 and $1.00 \mathrm{mg}$ protein/mL, respectively, both at $e P_{g}{ }^{50 P a}$ and $e P_{g}^{200 P a}$ (Table 1). The expansion rate is influenced by the nature of the stress-boundary conditions (Fig 1B) and the pressure difference between the center of the TF and its Plateau border regions, although both phenomena are related (Chatzigiannakis et al. 2020). 
Table 1. Drainage times, radii at rupture or 180 s after forcibly inducing drainage (i.e. equilibrium radius), expansion rates, bulk viscosities, equilibrium surface tensions, and/or Boussinesq numbers (see eq 7) of wheat and rye dough liquor and oat batter liquor thin films at lowered as well as at their native bulk concentrations. Abbreviations: $D L$, dough liquor; batter liquor, BL; TF, thin film; $\mathrm{s}^{-1}$, unit for the applied shear rate.

547

\begin{tabular}{|c|c|c|c|c|c|c|}
\hline & \multicolumn{2}{|c|}{ Wheat dough liquor } & \multicolumn{2}{|c|}{ Rye dough liquor } & \multicolumn{2}{|c|}{ Oat batter liquor } \\
\hline Lowered bulk concentration & \multicolumn{2}{|c|}{$1.50 \mathrm{mg}$ protein $/ \mathrm{mL}$} & \multicolumn{2}{|c|}{$0.50 \mathrm{mg}$ protein $/ \mathrm{mL}$} & \multicolumn{2}{|c|}{$1.00 \mathrm{mg}$ protein $/ \mathrm{mL}$} \\
\hline DL/BL bulk viscosity (mPa.s) & \multicolumn{2}{|c|}{$1.12 \pm 0.02$} & \multicolumn{2}{|c|}{$1.09 \pm 0.01$} & \multicolumn{2}{|c|}{$1.06 \pm 0.01$} \\
\hline $\mathrm{DL} / \mathrm{BL}$ equilibrium surface tension $(\mathrm{mN} / \mathrm{m})$ & \multicolumn{2}{|c|}{$46 \pm 1$} & \multicolumn{2}{|c|}{$48 \pm 1$} & \multicolumn{2}{|c|}{$35 \pm 1$} \\
\hline Excess gas pressure $(\mathrm{Pa})$ & $\underline{50}$ & $\underline{200}$ & $\underline{50}$ & $\underline{200}$ & $\underline{50}$ & $\underline{200}$ \\
\hline TF drainage time (s) & $8 \pm 1$ & $7 \pm 1$ & $9 \pm 1$ & $6 \pm 1$ & $13 \pm 1$ & $8 \pm 2$ \\
\hline TF Radius at rupture $(\mu \mathrm{m})$ & $156 \pm 40$ & $439 \pm 28$ & $331 \pm 41$ & $702 \pm 29$ & $369 \pm 42$ & $649 \pm 64$ \\
\hline TF expansion rate $(\mu \mathrm{m} / \mathrm{s})$ & $7 \pm 3$ & $30 \pm 6$ & $17 \pm 4$ & $61 \pm 10$ & $12 \pm 2$ & $41 \pm 12$ \\
\hline TF Boussinesq number (-) & $1401 \pm 322$ & $475 \pm 30$ & $873 \pm 117$ & $407 \pm 19$ & $26 \pm 3$ & $15 \pm 1$ \\
\hline Native bulk concentration & \multicolumn{2}{|c|}{$40.1 \mathrm{mg}$ protein $/ \mathrm{mL}$} & & & \multicolumn{2}{|c|}{$7.0 \mathrm{mg}$ protein $/ \mathrm{mL}$} \\
\hline $\mathrm{DL} / \mathrm{BL}$ bulk viscosity (mPa.s) & \multirow{2}{*}{\multicolumn{2}{|c|}{$\begin{array}{c}62\left(\text { at } 1 \mathrm{~s}^{-1}\right)-6\left(\text { at } 100 \mathrm{~s}^{-1}\right) \\
42 \pm 1\end{array}$}} & & & \multirow{2}{*}{\multicolumn{2}{|c|}{$\begin{array}{c}6\left(\text { at } 1 \mathrm{~s}^{-1}\right)-2\left(\text { at } 100 \mathrm{~s}^{-1}\right) \\
32 \pm 1\end{array}$}} \\
\hline $\mathrm{DL} / \mathrm{BL}$ equilibrium surface tension $(\mathrm{mN} / \mathrm{m})$ & & & & & & \\
\hline Excess gas pressure $(\mathrm{Pa})$ & $\underline{50}$ & $\underline{200}$ & & & $\underline{50}$ & $\underline{200}$ \\
\hline TF drainage time (s) & $>180$ & $>180$ & & & $>180$ & $>180$ \\
\hline TF equilibrium radius $(\mu \mathrm{m})$ & $552 \pm 70$ & $830 \pm 8$ & & & $583 \pm 157$ & $768 \pm 99$ \\
\hline TF expansion rate $(\mu \mathrm{m} / \mathrm{s})$ & $5 \pm 1$ & $18 \pm 1$ & & & $3 \pm 1$ & $14 \pm 1$ \\
\hline
\end{tabular}



$e P_{g}^{50 P a}$ (supplementary information) and $e P_{g}^{200 P a}$ (Fig 3), while those surrounding rye $\mathrm{DL}$ and oat $\mathrm{BL}$ TFs at 0.50 and $1.00 \mathrm{mg}$ protein/mL, respectively, were deformed into a dimple after applying $e P_{g}^{50 P a}$ (only oat BL TFs) (supplementary information) and $e P_{g}^{200 P a}$ (both rye DL and oat BL TFs) (Figs 4 and $\mathbf{5}$ ). These two observations suggest that A-W interfaces of wheat DL TFs at $1.50 \mathrm{mg}$ protein/mL have a higher stress carrying capacity than those surrounding rye DL and oat BL TFs at 0.50 and $1.00 \mathrm{mg}$ protein/mL, respectively. That the Bq numbers (Eq 7) of wheat DL TFs at $1.50 \mathrm{mg}$ protein $/ \mathrm{mL}$ at $e P_{g}{ }^{50 P a}$ and $e P_{g}{ }^{200 P a}$ were higher than the corresponding Bq numbers of rye DL and oat BL TFs at 0.50 and 1.00 mg protein/mL, respectively, supports this view (Table 1). Indeed, a higher Bq number implies a greater contribution of the surface stress-boundary conditions (Hermans et al. 2015). oat BL stabilized interfaces at lowered bulk concentrations, all these DL TFs still had similar drainage times. Two phenomena may be at the basis for this. First, in most cases drainage occurs faster when the TF radius is lower (Leal, 2004; Janssen and Anderson, 2011). Second, liquid drains more rapidly from between two planar than from between two dimpled A-W interfaces (Fig 1A) (Joye et al. 1992). and oat $\mathrm{BL}$ TFs at $1.00 \mathrm{mg}$ protein/mL rapidly reached thicknesses at which their stability mostly depends on the magnitude of $\Pi$. That rupture occurred at TF thicknesses of $20 \mathrm{~nm}$ for wheat and rye DL TFs and $12 \mathrm{~nm}$ for oat BL TFs implies that at this point the combined contributions of $P_{c}$ and $\Pi_{V D W}$ were larger than those of $\Pi_{e l}$ and $\Pi_{s t}($ Fig $\mathbf{1 C})$, even though for instance for rye, it was argued earlier that adsorbed proteins indeed engage in intermolecular interactions. It is possible that rupture may be too low to counteract those of $P_{c}$ and $\Pi_{v D w}$. 
572 liquor thin films at their native concentrations

573

574

575

576

577

578

579

580

581

582

583

584

585

586

587

588

589

590

591

592

593

\subsubsection{Thin film structural changes}

\section{WHEAT DOUGH LIQUOR}

Fig 6 shows interferometry images of wheat DL TFs at their native concentration at different time points after applying $e P_{g}{ }^{200 P a}(\mathbf{A}$ to $\mathbf{D}$ ). The 3D pixel intensity plot of Fig 6D (i.e. the image taken $180 \mathrm{~s}$ after drainage was forcibly induced) is shown in Fig 6E. After applying $e P_{g}{ }^{200 P a}$, the A-W interfaces surrounding wheat DL TFs at their native concentrations were deformed into a dimple (Figs 6A). Wheat DL constituents (visually observed as black and white spots in Fig 6A) were observed to migrate to the center of the dimple. As mentioned in section 1, such behavior illustrates that wheat DL constituents seemed to exert Marangoni stresses. This implies that the constituents adsorbed at wheat DL TF A-W interfaces do not form a continuous viscoelastic protein network and that lipids dominate A-W interfaces of wheat DL TFs at their native concentration. Dimple formation was followed by it being 'washed out' from the TF into the Plateau border region (Figs 1A and 6B). Once the dimple had been washed out, three distinct regions were observed in wheat DL TFs at their native concentration: a white region (labeled $h_{1}$ in Figs $6 \mathrm{C}$ and $6 \mathrm{E}$ ), a dark grey region (labeled $h_{2}$ in Figs $6 \mathrm{C}$ and $\mathbf{6 E}$ ), and black spots embedded in this dark grey region (indicated by white arrows in Fig $6 \mathrm{C}$ ). This is further illustrated by the red/green and dark blue regions, respectively, in the corresponding 3D pixel intensity plots (Fig 6E). After $180 \mathrm{~s}$, the white and dark grey regions had average $h_{\text {eq }}$ values of 79 \pm 4 and $24 \pm 2 \mathrm{~nm}$, respectively (Fig 6D). Although it was not possible to determine $h_{e q}$ of the black spots embedded in the dark grey region accurately, their thickness is expected to be lower than $24 \mathrm{~nm}$ (Fig 6C). In addition, two dark black circles representing remnants of the dimple were observed (Figs 6C, 6D, and 6E). 
Dimpled air-water interfaces

Plateau border
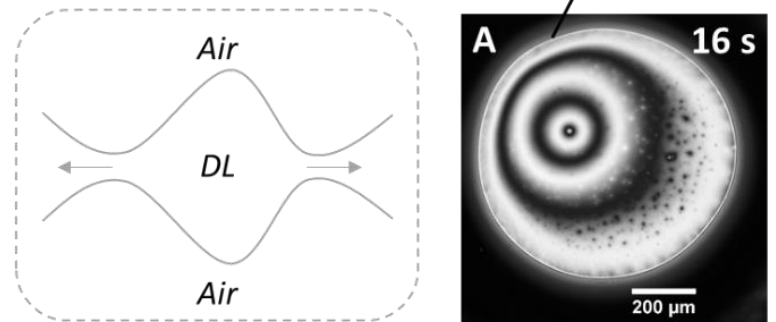

Symmetric dimple

Marangoni stresses

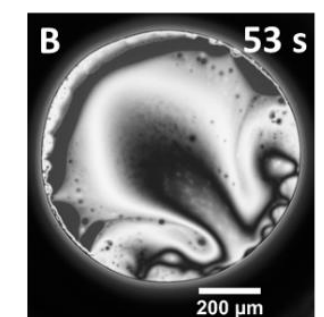

Dimple wash-out
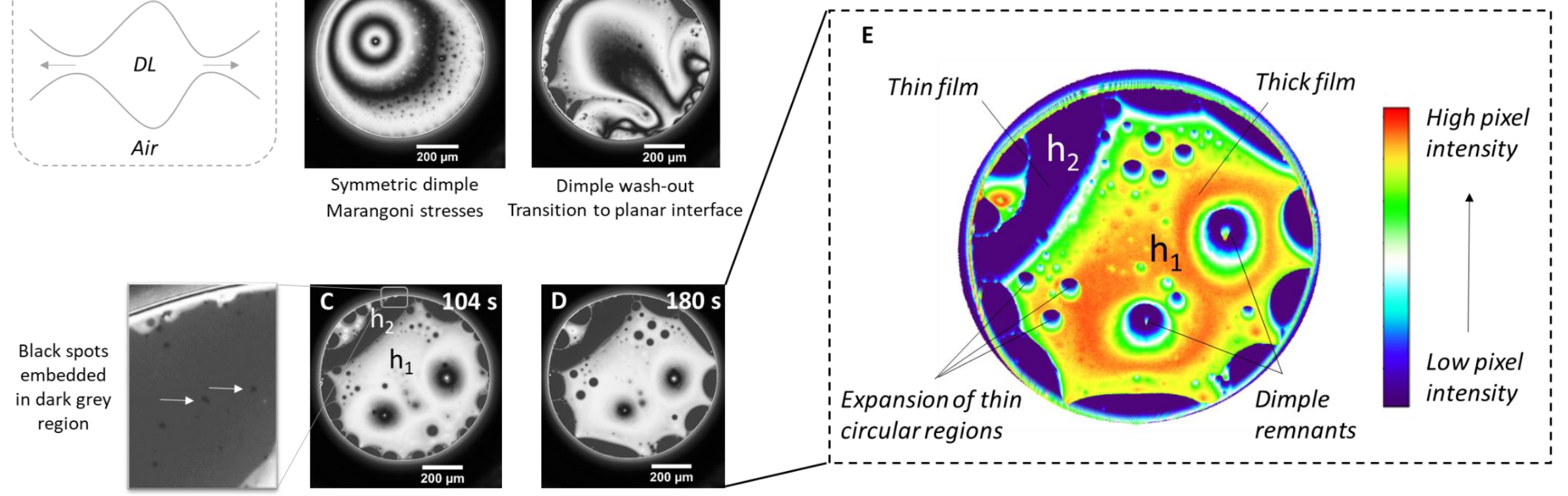

Stratification or step-wise drainage of supramolecular

structures (likely micelles) through the expansion of

visually darker and thus thinner circular domains

Fig 6. Interferometry images of wheat dough liquor (DL) thin films (TFS) at their native concentration at different time points after applying an excess gas

pressure of $200 \mathrm{~Pa}\left(\mathrm{eP}_{\mathrm{g}}^{200 \mathrm{2} a}\right)$. D represents the image $180 \mathrm{~s}$ after applying $\mathrm{eP}_{\mathrm{g}}^{\text {2000a }}$ and is presented in E as a 3D intensity plot, in which lower TF pixel intensities 
Over time, dark grey circular domains (dark blue circular regions in Fig 6E), which expanded and

600 merged with other similar domains, emerged in the white region (Figs 6C, 6D, and 6E). These domains had

601 similar $h_{e q}$ values as the larger dark grey region surrounding the white region. The white regions most

602 likely represent a thick film containing wheat DL constituents separated by two interfacial lipid

603 monolayers. The increase in area of thinner dark grey regions at the expense of thicker white regions (Figs

$6046 C, 6 D$, and $6 E$ ) is a phenomenon known as stratification. Stratification occurs when a TF contains at least

605 two layers of supramolecular structures (Nikolov and Wasan, 1989; Kralchevski et al. 1990; Bergeron et

606 al. 1992; Sonin and Langevin, 1993). Precisely because of this layered structuring, stratifying TFs thin in a

607 stepwise fashion. The $\Delta h_{e q}$ value of each such step $\left(\Delta h_{e q, s t e p}\right)$ can be estimated by calculating the sum of (i)

608 the characteristic length $(/)$ of the supramolecular structures confined in the TF and (ii) the Debye length

$609(1 / \kappa)$, which is a measure for the distance between different layers (Nikolov and Wasan, 1989):

610

$$
\Delta h_{\text {eq, step }}=2\left(l+\frac{1}{\kappa}\right)(\mathbf{E q} \text { 12) }
$$

That stratification proceeded through the growth of circular domains indicates that the 612 supramolecular structures in wheat DL TFs are lipid micelles (Nikolov and Wasan, 1989). This agrees well

613 with the observations that Marangoni effects are at play at the interfaces (see section 1 ) and that wheat

$614 \mathrm{DL}$ at its native concentration was above the critical micelle concentration (see supplementary

615 information). The radius of lipid micelles largely corresponds to the length of the hydrocarbon chains of 616 the participating lipid molecules (Reiss-Husson and Luzzati, 1964). Linoleic acid is the most abundant FA

617 in wheat DL lipid species (Janssen et al. 2020c). The characteristic length of a single linoleic acid molecule

618 was calculated ${ }^{1}$ to be $2.04 \mathrm{~nm}$ which implies that the radius of linoleic acid micelles would approximately 619 be $4.08 \mathrm{~nm}$. This value agrees well with an $h_{e q}$ value of $3.91 \mathrm{~nm}$ reported by Beltramo, Van Hooghten and 620 Vermant (2016) for phospholipid bilayers.

\footnotetext{
${ }^{1}$ The characteristic length of a single linoleic acid molecule was calculated by summing up the lengths of the different carbon-carbon bonds, considering bond angles and double bond cis configurations.
} 
The Debye length $(1 / \kappa)$ can be calculated as follows (Nikolov and Wasan, 1989):

$$
\kappa^{2}=\frac{4 \pi e^{2} N_{A}}{\varepsilon k T} C_{e l}(\text { Eq 13) }
$$

with $e$ the charge of an electron $\left(4.80 \times 10^{-10}\right.$ statcoulomb or $\left.\mathrm{cm}^{3 / 2} \mathrm{~g}^{1 / 2} \mathrm{~s}^{-1}\right), N_{A}$ Avogadro's number

$624\left(6.02 \times 10^{23} \mathrm{~mol}^{-1}\right), \varepsilon$ the dielectric constant of the bulk phase, $k$ the Boltzmann constant $\left(1.38 \times 10^{-23} \mathrm{~m}^{2}\right.$ $\left.625 \mathrm{~kg} \mathrm{~s}^{-2} \mathrm{~K}^{-1}\right), T$ the temperature $(298.15 \mathrm{~K})$, and $C_{e l}$ the total concentration of electrolytes in the bulk phase

626 (in $\left.\mathrm{mol} / \mathrm{m}^{3}\right)$. Somewhat unfortunately, DL contains a multitude of electrolytes such as endogenously 627 present minerals, sodium chloride, proteins, as well as free FAs and phospholipids (assuming that they 628 occur in their dissociated forms). In addition, the present proteins, free FAs, and phospholipids have a 629 great structural heterogeneity, with molecules varying considerably in molecular weight and charge 630 density. Thus, it is impossible to determine the exact electrolyte concentration (in $\mathrm{mol} / \mathrm{m}^{3}$ ) in DL and even 631 to estimate it would be at risk of making a fairly large error in calculating the value of $\Delta h_{\text {eq,step }}$ (Eq 12). 632 Nonetheless, assuming that the diameter of the lipid micelles in stratifying wheat DL TFs is approximately $6334.08 \mathrm{~nm}$ and that a lipid monolayer exists at both TF A-W interfaces, it is reasonable to state that the dark 634 blue regions, with an $h_{e q}$ of $24 \mathrm{~nm}$, in Fig $6 \mathrm{E}$ contain at least one layer of micelles. Moreover, this would 635 imply that the earlier mentioned black spots, with an estimated $h_{e q}$ lower than $24 \mathrm{~nm}$, embedded in the 636 dark grey region (Fig 6 C) represent either one layer of micelles or merely an inverse lipid bilayer or 637 common black film.

\section{OAT BATTER LIQUOR}

Fig 7 shows interferometry images of oat BL TFs at their native concentration at different time 640 points after applying $e P_{g}^{200 P a}(A$ to $\mathbf{D})$. The 3D pixel intensity plot of Fig 7D (i.e. the image taken $180 \mathrm{~s}$ after 641 drainage was forcibly induced) is shown in Fig 7E. Interestingly, while surface mobility was observed 642 during drainage of oat BL TFs at lowered bulk concentrations (see section 3.2.1.1), this was not the case 643 during drainage of oat BL TFs at their native concentration. 


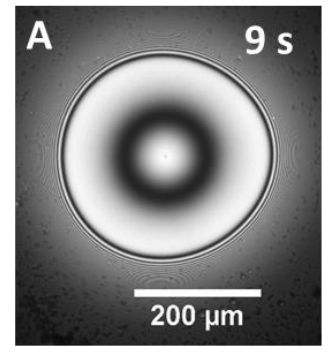

Symmetric dimple

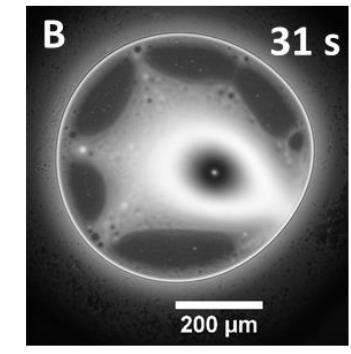

Dimple wash-out Transition to planar interface
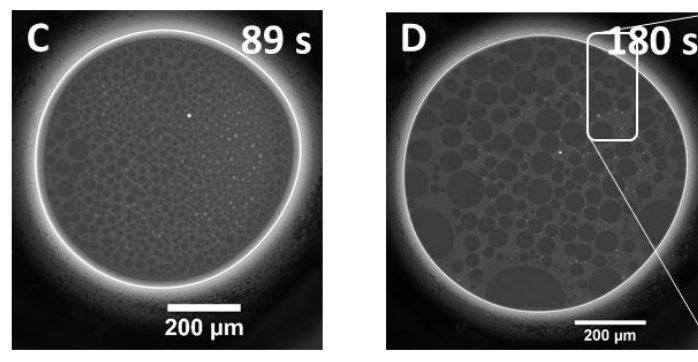

Stratification or step-wise drainage of supramolecular structures (likely micelles) through the expansion of visually darker and thus thinner circular domains
644

645

646

647

Fig 7. Interferometry images of oat batter liquor (BL) thin films (TFs) at their native concentration at different time points after applying an excess gas pressure of $200 \mathrm{~Pa}\left(\mathrm{eP}_{\mathrm{g}}^{200 \mathrm{~Pa}}\right)$. D represents the image $180 \mathrm{~s}$ after applying $\mathrm{PP}_{\mathrm{g}}{ }^{200 \mathrm{~Pa}}$ and is presented in $\mathrm{E}$ as a $3 \mathrm{D}$ intensity plot, in which lower TF pixel intensities correspond to a lower equivalent thickness $\left(h_{\text {eq }}\right)$. 
This most likely relates to the fact that at higher bulk concentrations diffusion of lipids from the

649 bulk phase to the $\mathrm{A}-\mathrm{W}$ interface rapidly (in less than $10 \mathrm{~ms}$, which was the maximal temporal resolution

650 of the camera, see section 2.2.4) restored interfacial concentration (and thus $\sigma$ ) gradients, thereby

651 suppressing otherwise occurring Marangoni stresses. Similar results have been observed for surfactant

652 films at concentrations exceeding the critical micelle concentration (Radoev, Dimitrov and Ivanov, 1974;

653 Bhamla Saad, Chai, Alvarez-Valenzuela, Tajuelo and Fuller, 2017). It thus seems that A-W interfaces of oat

654 BL TFs at their native concentration consist of densely-packed lipid monolayers. The low equilibrium $\sigma$ of

655 A-W interfaces stabilized by constituents in oat BL at its native concentration (Table 1 ) supports this.

656

For oat $\mathrm{BL}$ TFs at $e P_{g}{ }^{200 P a}$ at their native concentration, three distinct regions were observed after

657 the dimple had washed out: small white spots (Fig 7C, 7D, and 7E), light grey regions (labeled $h_{1}$ in Figs 7D

658 and 7E), and dark grey regions (labeled $h_{2}$ in Figs 7D and 7E). The white spots had $h_{e q}$ values ranging from

65920 to $60 \mathrm{~nm}$ (Fig 7D) and appeared as discrete dark red entities in the corresponding 3D pixel intensity

660 plots (Fig 7E). Such spots have been referred to as 'satellite fluid pockets' or 'Rayleigh-type instabilities'

661 (Bergeron et al. 1992) and nanoscopic mesas or terraces (Zhang et al. 2016) ${ }^{2}$ and are believed to originate

662 from accumulation of surface active moeities at the rim of the expanding domains. This rim breaks apart

663 as a result of local differences in $\Pi_{\text {sup }}$ (Fig $1 \mathrm{C}$ ) as well as in curvature and thus in $P_{L}$ between its interior and

664 exterior (Zhang et al. 2016). At $e P_{g}{ }^{200 P a}$, the light and dark grey regions had average $h_{\text {eq }}$ values of $18 \pm 2$

665 and $15 \pm 2 \mathrm{~nm}$, respectively (Figs 7D), which corresponded to high (light red) and low (green) TF pixel

666 intensities in Fig 7E.

667

That thinner circular regions expanded at the expense of thicker regions shows that oat BL TFs at

668 their native concentration - as did wheat DL TFs - exhibited stratification due to layering of micelles. This

669 is in line with the presence of a lipid interfacial monolayer (see section 3.2.1.1) and with $\sigma$ remaining

\footnotetext{
${ }^{2}$ Bergeron et al. (1992) and Zhang et al. (2016) compared these instabilities with the rim of a volcano crater that disintegrates into discrete pockets.
} 
constant already at an oat BL bulk concentration of $0.10 \mathrm{mg}$ protein $/ \mathrm{mL}$ (see supplementary information). A similar reasoning as above for wheat DL TFs results in an estimated oat BL TF lipid micelle diameter of approximately $4.08 \mathrm{~nm}$. Hence, assuming that there is a lipid monolayer at both A-W interfaces, it seems likely that the light red planar regions, with an $h_{e q}$ of $18 \mathrm{~nm}$, in Fig 7E may contain at least one layer of micelles, whilst the green regions, with an $h_{e q}$ of $15 \mathrm{~nm}$, in Fig 7E may represent merely an inverse lipid bilayer or common black film.

\subsubsection{Thin film stability}

Neither at $e P_{g}^{50 P a}$ nor at $e P_{g}{ }^{200 P a}$ did wheat $\mathrm{DL}$ and oat $\mathrm{BL}$ TFs at their native concentrations rupture after $180 \mathrm{~s}$ (Table 1). Some additional drainage experiments at $e P_{g}^{50 P a}$ showed that neither of the TFs ruptured even after $600 \mathrm{~s}$. Both wheat $\mathrm{DL}$ and oat BL TFs at their native concentrations were thus very stable. Despite similar TF stabilities, some notable differences in the wheat DL and oat BL TF drainage dynamics were observed.

Although wheat DL and oat BL TFs at their native concentrations expanded to a similar $R_{T F, e q}$ over time after applying $e P_{g}{ }^{200 P a}$, oat BLTFs expanded slower than wheat DL TFs, despite the higher $\eta_{\mathrm{b}}$ and $P_{L, b w}$ (Eq 3) of the latter (Table 1). This discrepancy may to some extent relate to Marangoni stresses during drainage of the latter but not during that of the former. Indeed, Marangoni stresses may create a line tension gradient between the rim of the dimple (higher concentration of surface-active constituents) and the TF Plateau border region (lower concentration of surface-active constituents) (Ivanov and Kralchevsky, 1997). Such gradient is expected to promote the diffusion of constituents towards the Plateau border region, which may increase the TF expansion rate.

Another notable difference in wheat and oat BL TF drainage dynamics was that, irrespective of the applied $e P_{g}$, the dimple of a wheat DL TF had a greater thickness at its center than an oat BL TF dimple

(Figs 6A and 7A). This implies a higher stress-carrying capacity for wheat DL TF than for oat BL TF A-W interfaces, which is in line with the observation that surface mobility by lipids, and thus the occurrence of 
Marangoni forces, was only observed during drainage of the former. That interfaces with greater stress-

695 carrying capacities result in larger dimples has also been noted elsewhere (Dai and Leal, 2008; Janssen and Anderson, 2011).

Interestingly, wheat DL TF dimples washed out later (51 $\pm 2 \mathrm{~s}$ after applying $\mathrm{eP}_{\mathrm{g}}{ }^{200 \mathrm{~Pa}}$ ) than those

698 of oat BL TFs (29 $\pm 4 \mathrm{~s}$ after applying $\mathrm{eP}_{\mathrm{g}}{ }^{200 \mathrm{~Pa}}$ ) (Figs 6B and 7B). In this regard, Joye et al. (1994) for a TF consisting of sodium dodecyl sulfate (SDS) or SDS-1- dodecanol (100:1) demonstrated that the magnitude of the interfacial shear viscosity $\left(\left|\eta_{s}\right|\right)$ largely determines the moment at which dimpled A-W interfaces 701 are washed out from the TF. It would thus be of interest to assess the interfacial shear rheology of wheat $702 \mathrm{DL}$ and oat $\mathrm{BL}$ at their native concentrations. However, the exact mechanism driving the wash-out of dimples from TFs is still not well-understood. Finally, it remains to be investigated whether dimpling would even occur in bread making as gas cells in dough expand quite slowly and the TFs separating them may not experience an abrupt $P_{H}$ increase.

Earlier it was shown that wheat $\mathrm{DL}$ and oat BL TFs at their native concentrations display stratification behavior. Stratifying TFs have high stability as they are thermodynamically metastable. Indeed, each number $(>1)$ of micellar layers creates an additional metastable state in the $\Pi$ isotherm 709 (Nikolov and Wasan, 1989; Bergeron and Radke, 1992) (Fig 1C). The magnitude of $\Pi_{\text {sup }}$ at these metastable TF thicknesses generally increases as the number of micellar layers decreases and can reach values up to $711200 \mathrm{~Pa}$ (Zhang and Sharma, 2018) or even 1,000 Pa (Basheva et al. 2007). However, the magnitude of $\Pi_{\text {sup }}$ 712 at such metastable thicknesses is system-specific and amongst others depends on the molecular structure 713 and concentration of the involved surfactants (Basheva et al. 2007; Zhang et al. 2016). Nonetheless, earlier 714 it was mentioned that gas cells in bread dough at the end of fermentation may experience $P_{L, \text { gas cell }}$ values 715 lower than $200 \mathrm{~Pa}$. It thus seems reasonable to assume that at this stage at least some of the gas cells 716 which are separated only by a TF are metastable and therefore temporarily prevented from coalescing. 717 Furthermore, even at low TF thicknesses $(<30 \mathrm{~nm})$ wheat DL and oat BL TFs at their native concentrations 
remained stable. This implies that there may have been a contribution of $\Pi_{e l}$ and/or $\Pi_{s t}$ to the total $\Pi$ of said TFs, possibly originating from intermolecular interactions ocurring between the lipid monolayers at both TF A-W interfaces.

\subsection{Implications for bread making research}

Based on the above, it can be concluded that the stability of gas cells during bread dough fermentation and early baking likely not only depends on the characteristics of their A-W interfaces, but also on those of the TFs separating them. Indeed, the use of a pressure-controlled dynamic TF balance can provide beyond state-of-the-art insights into the potential of DL constituents to stabilize gas cells in bread making. Moreover, the present study demonstrates the importance of studying the drainage dynamics of DL TFs at native rather than at lowered concentrations, as the bulk and interfacial stabilization mechanisms in both cases were substantially different. Wheat $\mathrm{DL}$ and oat $\mathrm{BL}$ constituents when assessed at their native concentration may contribute substantially to stabilizing gas cells in dough and thus likely impact bread loaf volume and crumb structure. That wheat breads have a considerably higher loaf volume than oat breads (Janssen et al. 2018) is mostly due to the viscoelastic gluten network which by displaying strain hardening acts as the primary gas cell stabilizing force (van Vliet et al. 1992). Thus, assuming that oat batter aqueous phase constituents contribute to gas cell stability in oat bread making, they cannot match the efficiency of the combined contributions of the gluten network and dough aqueous phase constituents in terms of stabilizing gas cells in wheat bread making.

\section{Conclusions}

The role of TF drainage dynamics in determining gas cell stability in bread making has so far received little attention. Most available experimental studies dealing with the $A-W$ interfacial properties of DL/BL constituents have thus far been carried out at concentrations much below those in real 
dough/batter systems. In the present work, we studied DL/BL TFs at their native concentrations, or at least at concentrations as high as possible without clogging the microfluidic chips. First, DL/BL TFs at their native concentrations expanded slower and to a larger radius than those at lowered concentrations. This was related to differences in the nature of the stress-boundary conditions, $\eta_{b}$ (although at both concentrations NSPs were enzymatically hydrolyzed) and pressure differences between the TF center and Plateau border, which are interrelated phenomena. Weakly viscoelastic A-W interfacial layers dominated by proteins were characteristic of rye DL TFs at $0.50 \mathrm{mg}$ protein $/ \mathrm{mL}$ (Fig $8 \mathrm{C1}$ ). Wheat DL TFs at relatively low concentrations ( $1.50 \mathrm{mg}$ protein $/ \mathrm{mL}$ ) were characterized by weakly viscoelastic mixed protein-lipid AW interfaces (Fig 8 A1). In contrast, wheat DL TF A-W interfaces at their native concentration seemed to be dominated by lipids which then generated Marangoni stresses upon formation of a dimpled film (Fig 8 A2). Whilst dimples and likely thus the associated convective effects were also observed for oat BL TFs, both at $1.00 \mathrm{mg}$ protein/mL (Fig 8 B1) and at native concentration (Fig 8 B2), surface mobility by lipids was only observed at the former concentration. Second, the dimpling for wheat DL and oat BL TFs at native concentration likely also contributed to their reduced liquid drainage rates. Finally, DL/BL TFs at their native concentration seemed to be stable at low TF thicknesses, due to a combined effect of stratification and intermolecular repulsive forces, whilst at lowered bulk concentrations they were not. We conclude that wheat $\mathrm{DL}$ and oat $\mathrm{BL}$ constituents when assessed at their native concentration might contribute substantially to stabilizing gas cells in dough and thus likely also to bread loaf volume and crumb structure. 
A1

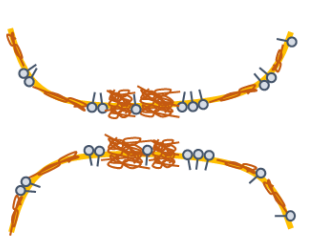

Stress-poor A-W interfacial films

$$
\text { Planar shape }
$$

No contribution of $\Pi_{\text {sup }} \Pi_{e l}$ or $\Pi$

A2

Bulk concentration equal to that in the supernatant after ultracentrifugation
758

759

760

761

762

763

764

765
B1

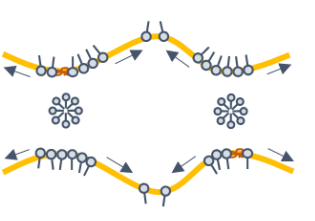

Stress-carrying A-W interfacial films Dimpled shape

Small contribution of $\Pi_{\text {sup }}$

B2
C1

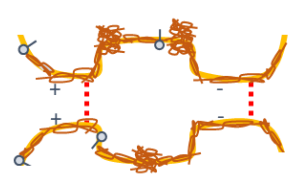

Stress-poor A-W interfacial films Dimpled shape Small contribution of $\Pi_{e l}$ and/or $\Pi_{\text {st }}$

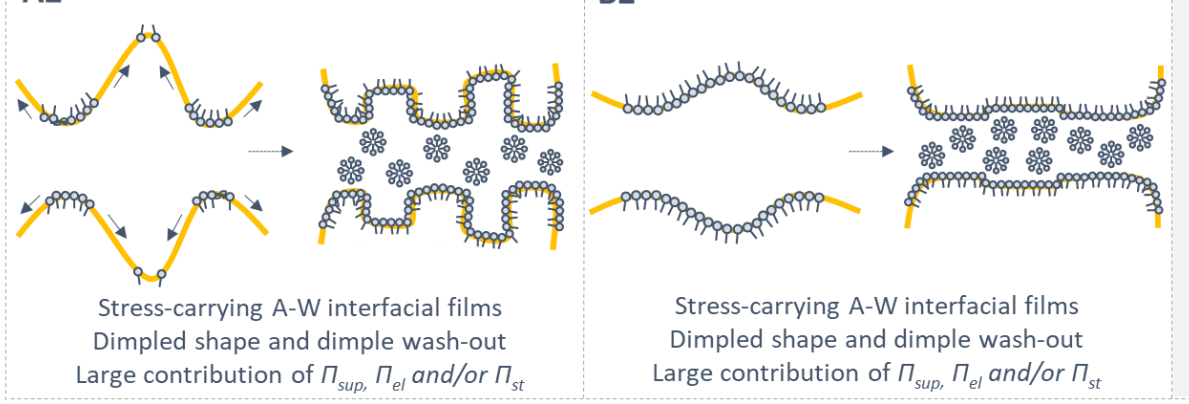

\& Protein

Protein aggregate

Lipid

ㅇํㅇㅇำ Micelle

Fig 8. Schematic overview of the structure of wheat dough liquor (DL) (A1), oat batter liquor (BL) (B1), and rye DL (C1) thin films (TFs) at 1.50, 1.00, and $0.50 \mathrm{mg}$ dry matter protein/mL, respectively, as well as of wheat DL (A2) and oat (B2) BL TFs at their native concentrations (i.e. at concentrations equal to those occurring in the respective supernatant after ultracentrifugation). Rye DL TFs at their native concentration could not be monitored because rye $D L$ constituents formed large aggregates in the microchip channels which prevented drainage to occur freely. Abbreviations: $\Pi_{\text {sup }}$ disjoining pressure contribution from oscillatory structural forces; $\Pi_{e l}$, disjoining pressure contribution from electrostatic repulsive forces; $\Pi_{\text {st }}$ disjoining pressure contribution from steric repulsive forces; $A-W$, air-water. 


\section{Acknowledgements}

767

768

769

770

771

\section{References}

773

774

775

776

777

778

779

780

781

782

783

784

785

786 30.

Frederik Janssen and Arno G.B. Wouters gratefully acknowledge the Research Foundation Flanders (FWO - Vlaanderen, Brussels, Belgium) for positions as doctoral (project grant SB151453 and travel grant V418319N) and postdoctoral (grant 12V6118N) researchers, respectively. Jan A. Delcour is W.K. Kellogg Chair in Cereal Science and Nutrition at KU Leuven. This work is part of the Methusalem program "Food for the Future".

Babin, P., Della Valle, G., Chiron, H., Cloetens, P., Hoszowska, J., Pernot, P., Réguerre, A. L., Salvo, L. and Dendievel, R. 2006. Fast X-ray tomography analysis of bubble growth and foam setting during breadmaking. Journal of Cereal Science 43(3), 393-397.

Baker, J. C., Parker, H. K. and Mize, M. D. 1946. Supercentrifugates from dough. Cereal Chemistry 23, 16-

Basheva, E. S., Gurkov, T. D., Christov, N. C. and Campbell, B. 2006. Interactions in oil/water/oil films stabilized by $\beta$-lactoglobulin: role of the surface charge. Colloids and Surfaces A 282-283, 99-108.

Basheva, E. S., Kralchevsky, P. A., Danov, K. D., Ananthapadmanabhan, K. P. and Lips, A. 2007. The colloid structural forces as a tool for particle characterization and control of dispersion stability. Royal Society of Chemistry 9(38), 5183-5198.

Belitz, H. D., Grosch, W. and Schieberle, P. 2009. Cereals and cereal products (pp. 673-746). Food Chemistry. Berlin, Germany: Springer-Verlag.

Beltramo, P. J., Van Hooghten, R. and Vermant, J. 2016. Millimeter-area, free standing, phospholipid bilayers. Soft Matter 12(19), 4324-4331. 
Beltramo, P. J. and Vermant, J. 2016. Simple optical imaging of nanoscale features in free-standing films. ACS omega 1(3), 363-370.

Bergeron, V., Jimenez-Laguna, A. and Radke, C. 1992. Hole formation and sheeting in the drainage of thin liquid films. Langmuir 8(12), 3027-3032.

Bergeron, V. and Radke, C. 1992. Equilibrium measurements of oscillatory disjoining pressures in aqueous foam films. Langmuir 8(12), 3020-3026.

Bhamla, M. S., Chai, C., Alvarez-Valenzuela, M. A., Tajuelo, J. and Fuller, G. G. 2017. Interfacial mechanisms for stability of surfactant-laden films. PLOS ONE 12(5), e0175753.

Bligh, E. G. and Dyer, W. J. 1959. A rapid method of total lipid extraction and purification. Canadian Journal of Biochemistry and Physiology 37, 911-917.

Cascão Pereira, L. G., Johansson, C., Blanch, H. W. and Radke, C. J. 2001. A bike-wheel microcell for measurement of thin-film forces. Colloids and Surfaces $A$ 186(1), 103-111.

Cascão Pereira, L. G., Johansson, C., Radke, C. J. and Blanch, H. W. 2003. Surface forces and drainage kinetics of protein-stabilized aqueous films. Langmuir 19(18), 7503-7513.

Chatzigiannakis, E., Veenstra, P., ten Bosch, D. and Vermant, J. 2020. Mimicking coalescence using a pressure-controlled dynamic thin film balance. Soft Matter 16, 9410-9422.

Chen, M., Sala, G., Meinders, M. B. J., van Valenberg, H. J. F., van der Linden, E. and Sagis, L. M. C. 2017. Interfacial properties, thin film stability and foam stability of casein micelle dispersions. Colloids and Surfaces B 149, 56-63.

Chen, M., Feijen, S., Sala, G., Meinders, M. B. J., van Valenberg, H. J. F., van Hooijdonk, A. C. M. and van der Linden, E. 2018. Foam stabilized by large casein micelle aggregates: the effect of aggregate number in foam lamella. Food Hydrocolloids 74, 342-348.

Courtin, C. M. and Delcour, J. A. 2001. Relative activity of endoxylanases towards water-extractable and water-unextractable arabinoxylan. Journal of Cereal Science 33(3), 301-312. 
811 Dagastine, R. R., Manica, R., Carnie, S. L., Chan, D., Stevens, G. W. and Grieser, F. 2006. Dynamic forces between two deformable oil droplets in water. Science 313(5784), 210-213.

813 Dai, B. and Leal, L. G. 2008. The mechanism of surfactant effects on drop coalescence. Physics of Fluids 20(4), 040802.

Damodaran, S. 2005. Protein stabilization of emulsions and foams. Journal of Food Science 70(3), 54-66.

816

817

Delcour, J. A., Vanhamel, S. and De Geest, C. 1989. Physico-chemical and functional properties of rye nonstarch polysaccharides. I. colorimetric analysis of pentosans and their relative monosaccharide compositions in fractionated (milled) rye products. Cereal Chemistry 66, 107111.

Derjaguin, B. V. and Landau, L. 1941. Theory of the stability of strongly charged lyophobic sols and of the adhesion of strongly charged particles in solutions of electrolytes. Acta Physico Chemica URSS 14, 633.

Dimitrova, T. D., Leal-Calderon, F., Gurkov, T. D. and Campbell, B. 2001. Disjoining pressure vs thickness isotherms of thin emulsion films stabilized by proteins. Langmuir 17(26), 8069-8077.

Dobraszczyk, B. J. 1997. Development of a new dough inflation system to evaluate doughs. Cereal Foods World 42(7), 516-519.

Dubreil, L., Biswas, S. C. and Marion, D. 2002. Localization of puroindoline-a and lipids in bread dough using confocal scanning laser microscopy. Journal of Agricultural and Food Chemistry, 50, 60786085.

Dumay, E. M., Kalichevsky, M. T. and Cheftel, J. C. 1994. High-pressure unfolding and aggregation of betalactoglobulin and the baroprotective effects of sucrose. Journal of Agricultural and Food Chemistry 42(9), 1861-1868. 
Englyst, H. N. and Cummings, J. H. 1984. Simplified method for the measurement of total non-starch polysaccharides by gas-liquid chromatography of constituent sugars as alditol acetates. Analyst 109(7), 937-942.

Exerowa, D. and Kruglyakov, P. M. 1997. Foam and foam films: theory, experiment, application. Amsterdam, The Netherlands: Elsevier.

Finney, K. 1984. An optimized, straight-dough, bread making method after 44 years. Cereal Chemistry 61(1), 20-27.

Frankel, S. P. and Mysels, K. J. 1962. On the dimpling during the approach of two interfaces. The Journal of Physical Chemistry 66(1), 190-191.

Fuller, G. G. and Vermant, J. 2012. Complex fluid-fluid interfaces: rheology and structure. Annual Review of Chemical and Biomolecular Engineering 3, 519-543.

Gan, Z., Angold, R. E., Williams, M. R., Ellis, P. R., Vaughan, J. G. and Galliard, T. 1990. The microstructure and gas retention of bread dough. Journal of Cereal Science 12(1), 15-24.

Gan, Z., Ellis, P. R. and Schofield, J. D. 1995. Gas cell stabilisation and gas retention in wheat bread dough. Journal of Cereal Science 21(3), 215-230.

Gochev, G., Retzlaff, I., Exerowa, D. and Miller, R. 2014. Electrostatic stabilization of foam films from $\beta$ lactoglobulin solutions. Colloids and Surfaces A 460, 272-279.

Gochev, G. G., Ulaganathan, V., Retzlaff, I., Gehin-Delval, C., Gunes, D. Z., Leser, M., Kulozik, U., Miller, R. and Braunschweig, B. 2020. $\beta$-Lactoglobulin adsorption layers at the water/air surface: 4. Impact on the stability of foam films and foams. Minerals 10(7), 636.

Hager, A.-S., Bosmans, G. M. and Delcour, J. A. 2014. Physical and molecular changes during the storage of gluten-free rice and oat bread. Journal of Agricultural and Food Chemistry 62(24), 5682-5689.

Hermans, E., Saad Bhamla, M., Kao, P., Fuller, G. G. and Vermant, J. 2015. Lung surfactants and different contributions to thin film stability. Soft Matter 11(41), 8048-8057. 
Husband, F. A. and Wilde, P. J. 1998. The Effects of caseinate submicelles and lecithin on the thin film drainage and behavior of commercial caseinate. Journal of Colloid and Interface Science 205(2), 316-322.

Ivanov, I. B. and Kralchevsky, P. A. 1997. Stability of emulsions under equilibrium and dynamic conditions. Colloids and Surfaces A 128(1-3), 155-175.

Janssen, F., Wouters, A. G. B., Pauly, A. and Delcour, J. A. 2018. Relating the composition and air/water interfacial properties of wheat, rye, barley, and oat dough liquor. Food Chemistry 264, 126-134.

Janssen, F., Wouters, A. G.B., Meeus, Y., Moldenaers, P., Vermant, J. and Delcour, J. A. 2020a. The role of non-starch polysaccharides in determining the air-water interfacial properties of wheat, rye, and oat dough liquor constituents. Food Hydrocolloids 105, 105771.

Janssen, F. 2020b. The role of wheat, rye and oat dough aqueous phase constituents in bread making Leuven, KU Leuven.

Janssen, F., Wouters, A. G. B., Linclau, L., Waelkens, E., Derua, R., Dehairs, J., Moldenaers, P., Vermant, J. and Delcour, J. A. 2020c. The role of lipids in determining the air-water interfacial properties of wheat, rye, and oat dough liquor constituents. Food chemistry 319, 126565.

Janssen, P. J. A. and Anderson, P. D. 2011. Modeling film drainage and coalescence of drops in a viscous fluid. Macromolecular Materials and Engineering 296(3-4), 238-248.

Joye, J. L., Hirasaki, G. J. and Miller, C. A. 1992. Dimple formation and behavior during axisymmetrical foam film drainage. Langmuir 8(12), 3083-3092.

Joye, J.-L., Hirasaki, G. J. and Miller, C. A. 1994. Asymmetric drainage in foam films. Langmuir 10(9), 31743179.

Kannan, A., Shieh, I. C., Leiske, D. L. and Fuller, G. G. 2018. Monoclonal antibody interfaces: Dilatation mechanics and bubble coalescence. Langmuir 34(2), 630-638. 
Kralchevski, P., Nikolov, A., Wasan, D. T. and Ivanov, I. 1990. Formation and expansion of dark spots in stratifying foam films. Langmuir 6(6), 1180-1189.

Larsson, H. and Eliasson, A. C. 1996. Phase separation of wheat flour dough studied by ultracentrifugation and stress relaxation. i. Influence of water content. Cereal Chemistry 73(1), 18-24.

Leal, L. G. 2004. Flow induced coalescence of drops in a viscous fluid. Physics of Fluids 16(6), 1833-1851.

Li, W., Dobraszczyk, B. J., and Wilde, P. J. 2004. Surface properties and locations of gluten proteins and lipids revealed using confocal scanning laser microscopy in bread dough. Journal of Cereal Science, $39,403-411$.

Lin, G., Frostad, J. M. and Fuller, G. G. 2018. Influence of interfacial elasticity on liquid entrainment in thin foam films. Physical Review Fluids 3(11), 114001.

MacRitchie, F. 1976. Liquid phase of dough and its role in baking. Cereal Chemistry 53(3), 318-326.

Maldonado-Valderrama, J. and Langevin, D. 2008. On the difference between foams stabilized by surfactants and whole casein or $\beta$-casein. Comparison of foams, foam films, and liquid surfaces studies. The Journal of Physical Chemistry B 112(13), 3989-3996.

Manikantan, H. and Squires, T. M. 2020. Surfactant dynamics: hidden variables controlling fluid flows. Journal of Fluid Mechanics 892.

Marinova, K. G., Basheva, E. S., Nenova, B., Temelska, M., Mirarefi, A. Y., Campbell, B. and Ivanov, I. B. 2009. Physico-chemical factors controlling the foamability and foam stability of milk proteins: Sodium caseinate and whey protein concentrates. Food Hydrocolloids 23(7), 1864-1876.

Marinova, K. G., Gurkov, T. D., Velev, O. D., Ivanov, I. B., Campbell, B. and Borwankar, R. P. 1997. The role of additives for the behaviour of thin emulsion films stabilized by proteins. Colloids and Surfaces A 123-124, 155-167.

Marinova, K. G., Stanimirova, R. D., Georgiev, M. T., Alexandrov, N. A., Basheva, E. S. and Kralchevsky, P. A. 2014. Co-adsorption of the proteins beta-casein and BSA in relation to the stability of thin liquid 

Chemistry for Nanotechnology. Boca Raton, FL: CRC Press.

906

907

908

909

910

Mauritzen, C. M. and Stewart, P. R. 1965. The ultracentrifugation of doughs made from wheat flour. Australian Journal of Biological Sciences 18(1), 173-189.

McCleary, B. V. and Nurthen, E. 1986. Measurement of (1-3)(1-4)- $\beta$-D-glucan in malt, wort and beer. Journal of the Institute of Brewing 92(2), 168-173.

Melis, S. 2019. The role of wheat endogenous lipids and their enzymatically released hydrolysis products in bread making. Leuven, KU Leuven.

Messens, W., Van Camp, J. and Huyghebaert, A. 1997. The use of high pressure to modify the functionality of food proteins. Trends in Food Science \& Technology 8(4), 107-112.

Mills, E. N. C., Wilde, P. J., Salt, L. J. and Skeggs, P. 2003. Bubble formation and stabilization in bread dough. Food and Bioproducts Processing 81(3), 189-193.

Murray, B. S. 2007. Stabilization of bubbles and foams. Current Opinion in Colloid \& Interface Science 12(4), 232-241.

Nikolov, A. and Wasan, D. 1989. Ordered micelle structuring in thin films formed from anionic surfactant solutions: I. Experimental. Journal of Colloid and Interface Science 133(1), 1-12.

Obeid, S., Guyomarch, F., Francius, G., Guillemin, H., Wu, X., Pezennec, S., Famelart, M.-H., Cauty, C., Gaucheron, F. and Lopez, C. 2019. The surface properties of milk fat globules govern their interactions with the caseins: Role of homogenization and pH probed by AFM force spectroscopy. Colloids and Surfaces B 182, 110363.

Pauly, A., Pareyt, B., Fierens, E. and Delcour, J. A. 2014. Fermentation affects the composition and foaming properties of the aqueous phase of dough from soft wheat flour. Food Hydrocolloids 37, 221-228. 
Primo-Martín, C., Hamer, R. J. and de Jongh, H. H. J. 2006. Surface layer properties of dough liquor components: are they key parameters in gas retention in bread dough? Food Biophysics 1(2), 8393.

Radoev, B., Dimitrov, D. S. and Ivanov, I. 1974. Hydrodynamics of thin liquid films effect of the surfactant on the rate of thinning. Colloid and Polymer Science 252(1), 50-55.

Reiss-Husson, F. and Luzzati, V. 1964. The Structure of the micellar solutions of some amphiphilic compounds in pure water as determined by absolute small-angle X-ray scattering techniques. The Journal of Physical Chemistry 68(12), 3504-3511.

Reynolds, O. 1886. IV. On the theory of lubrication and its application to Mr. Beauchamp tower's experiments, including an experimental determination of the viscosity of olive oil. Philosophical transactions of the Royal Society of London (177), 157-234.

Rullier, B., Axelos, M. A. V., Langevin, D. and Novales, B. 2009. Beta-Lactoglobulin aggregates in foam films: correlation between foam films and foaming properties. Journal of Colloid and Interface Science 336(2), 750-755.

Rullier, B., Axelos, M. A. V., Langevin, D. and Novales, B. 2010. Beta-Lactoglobulin aggregates in foam films: effect of the concentration and size of the protein aggregates. Journal of Colloid and Interface Science 343(1), 330-337.

Sahi, S. S. 1994. Interfacial properties of the aqueous phases of wheat flour doughs. Journal of Cereal Science 20(2), 119-127.

Sahi, S. S. 2003. The interfacial properties of the aqueous phases of full recipe bread doughs. Journal of Cereal Science 37(2), 205-214.

Salt, L. J., Wilde, P. J., Georget, D., Wellner, N., Skeggs, P. K. and Mills, E. N. C. 2006. Composition and surface properties of dough liquor. Journal of Cereal Science 43(3), 284-292. 
Salt, L. J., González-Thuillier, I., Chope, G., Penson, S., Tosi, P., Haslam, R. P., Skeggs, P. K., Shewry, P. R. and Wilde, P. J. 2018. Intrinsic wheat lipid composition effects the interfacial and foaming properties of dough liquor. Food Hydrocolloids 75, 211-222.

Sarker, D. K., Wilde, P. J. and Clark, D. C. 1998. Enhancement of protein foam stability by formation of wheat arabinoxylan-protein crosslinks. Cereal Chemistry 75(4), 493-499.

Senée, J., Robillard, B. and Vignes-Adler, M. 1999. Films and foams of Champagne wines. Food Hydrocolloids 13(1), 15-26.

Sethumadhavan, G. N., Nikolov, A. and Wasan, D. 2001. Film stratification in the presence of colloidal particles. Langmuir 17(7), 2059-2062.

Sheludko, A. 1967. Thin liquid films. Advances in Colloid and Interface Science 1(4), 391-464.

Sonin, A. A. and Langevin, D. 1993. Stratification dynamics of thin films made from aqueous micellar solutions. Europhysics Letters 22(4), 271.

Sroan, B. S., Bean, S. R. and MacRitchie, F. 2009. Mechanism of gas cell stabilization in bread making. i. The primary gluten-starch matrix. Journal of Cereal Science 49(1), 32-40.

Stefan, J. 1874. Versuchü ber die scheinbere adhäsion sitzungsberichteder mathematischnaturwissenschaften klasse der kaiserlichen akademie der wissenschaften, II. Abteilung. Denkschriften der Kaiserlichen Akademie der Wissenschaften / MathematischNaturwissenschaftliche Classe 69, 713-735.

Toshev, B. V. and Ivanov, I. B. 1975. Thermodynamics of thin liquid films. Colloid and Polymer Science 253(7), 558-565.

Turbin-Orger, A., Boller, E., Chaunier, L., Chiron, H., Della Valle, G. and Réguerre, A. L. 2012. Kinetics of bubble growth in wheat flour dough during proofing studied by computed X-ray microtomography. Journal of Cereal Science 56(3), 676-683. 
972 Turbin-Orger, A., Babin, P., Boller, E., Chaunier, L., Chiron, H., Della Valle, G., Dendievel, R., Réguerre, A. L. and Salvo, L. 2015a. Growth and setting of gas bubbles in a viscoelastic matrix imaged by X-ray microtomography: the evolution of cellular structures in fermenting wheat flour dough. Soft Matter 11(17), 3373-3384.

Turbin-Orger, A., Della Valle, G., Doublier, J. L., Fameau, A.-L., Marze, S. and Saulnier, L. 2015b. Foaming and rheological properties of the liquid phase extracted from wheat flour dough. Food Hydrocolloids 43, 114-124.

Vakarelski, I. U., Manica, R., Tang, X., O’Shea, S. J., Stevens, G. W., Grieser, F., Dagastine, R. R. and Chan, D. Y. 2010. Dynamic interactions between microbubbles in water. Proceedings of the National Academy of Sciences 107(25), 11177-11182.

Valkovska, D. S., Danov, K. D. and Ivanov, I. B. 2002. Stability of draining plane-parallel films containing surfactants. Advances in Colloid and Interface Science 96(1-3), 101-129.

van Vliet, T., Janssen, A. M., Bloksma, A. H. and Walstra, P. 1992. Strain hardening of dough as a requirement for gas retention. Journal of Texture Studies 23(4), 439-460.

Verwey, E. J. W. and Overbeek, J. T. G. 1948. Theory of the stability of lyophobic colloids. Mineola, NY: Dover Publications.

Webber, G. B., Edwards, S. A., Stevens, G. W., Grieser, F., Dagastine, R. R. and Chan, D. Y. C. 2008. Measurements of dynamic forces between drops with the AFM: novel considerations in comparisons between experiment and theory. Soft Matter 4(6), 1270-1278.

Wilde, P. J., Rodríguez Niño, M. R., Clark, D. C. and Rodríguez Patino, J. M. 1997. Molecular diffusion and drainage of thin liquid films stabilized by bovine serum albumin-Tween 20 mixtures in aqueous solutions of ethanol and sucrose. Langmuir 13(26), 7151-7157.

Wilde, P. J. 2000. Interfaces: their role in foam and emulsion behaviour. Current Opinion in Colloid and Interface Science 5(3), 176-181. 
996 Zhang, Y., Yilixiati, S., Pearsall, C. and Sharma, V. 2016. Nanoscopic Terraces, Mesas, and Ridges in Freely 997 Standing Thin Films Sculpted by Supramolecular Oscillatory Surface Forces. ACS Nano 10(4), 4678$998 \quad 4683$.

999 Zhang, Y. and Sharma, V. 2018. Nanoridge formation and dynamics of stratification in micellar $1000 \quad$ freestanding films. Langmuir 34(3), 1208-1217.

1001 